\title{
Baryon clustering at the critical line and near the hypothetical critical point in heavy-ion collisions
}

\author{
Edward Shuryak and Juan M. Torres-Rincon (1) \\ Department of Physics and Astronomy, Stony Brook University, Stony Brook, New York 11794-3800, USA
}

(Received 5 October 2018; revised manuscript received 6 February 2019; published 1 August 2019)

\begin{abstract}
We study clustering of baryons at the freeze-out point of relativistic heavy-ion collisions. Using a WaleckaSerot model for the nucleon-nucleon $(N N)$ interaction we analyze how the modified critical $\sigma$ moderesponsible for the $N N$ attraction-allows for clustering of nucleons when the system is close to a possible critical point of QCD. We investigate clusters of few nucleons, and also the internal cluster configuration when the system is long lived. For realistic heavy-ion collisions we study to what extent light clusters, such as ${ }^{4} \mathrm{He}$, can be formed in several $\mathrm{fm} / c$, and perform the statistical analysis of proton cumulants and higher-order moments (skewness and kurtosis) for collisions at the beam energy scan of the Relativistic Heavy-Ion Collider.
\end{abstract}

DOI: 10.1103/PhysRevC.100.024903

\section{INTRODUCTION}

We start by contrasting known facts about high- and lowenergy heavy-ion collisions, after which we will define the phenomena to be discussed in this work.

By high-energy collisions we mean those at the Relativistic Heavy-Ion Collider (RHIC) full energy $\sqrt{s_{N N}} \approx 200 \mathrm{GeV}$ and at the Large Hadron Collider (LHC) $\sqrt{s_{N N}} \approx 2-8 \mathrm{TeV}$. In these cases the particle yields are very accurately described by the so-called "resonance gas model," assuming that all interactions between hadrons can be effectively treated as an ideal gas of all known resonances, as suggested by the BethUhlenbeck formula. As shown, e.g., in Ref. [1], the yields per degree of freedom are on the same thermal exponent, from the lightest species - pions, kaons, etc. — up to the baryons, hyperons, and their antiparticles, all the way up to light nuclei such as ${ }^{4} \mathrm{He}$. This trend is observed to hold over about nine decades. The lesson is that, at chemical freeze-out temperatures $T_{\text {ch }} \simeq 150 \mathrm{MeV}$ and at near-zero baryonic chemical potential $\mu_{B} \simeq 0$, the fireball is very well thermally equilibrated. Such high degree of equilibration undoubtedly is related with kinetic properties of the strongly coupled quark-gluon plasma (QGP), preceding the freeze-out stage of these collisions.

At low nonrelativistic collision energies, $\sqrt{s_{N N}}<1 \mathrm{GeV}$, creating nuclear matter with temperatures $T \simeq 10 \mathrm{MeV}$, one observes the so-called "multifragmentation" phenomena, production of a large variety of heavy fragments of colliding nuclei, with wide powerlike distributions. It is attributed to a nearby presence of a critical point, separating liquid nuclear matter from a gaslike phase. For a review see, e.g., [2]. The production of various nuclear fragments is not in equilibrium,

Published by the American Physical Society under the terms of the Creative Commons Attribution 4.0 International license. Further distribution of this work must maintain attribution to the author(s) and the published article's title, journal citation, and DOI. Funded by $S C O A P^{3}$. and is very sensitive to the relation between the temperature and time available for cluster formation. Those are also associated with rapidities close to those of the beams. This regime can be compared to that in atomic physics studying various out-of-equilibrium situations, for example "snow production" machines, operating in between water and ice phases.

Our paper focuses on an entirely different range of collision energies and rapidities. While they are called "low" compared to typical collider regimes, they are still related with much higher temperatures. The lowest one, corresponding to "kinetic freeze-out", is still $T_{f} \sim 100 \mathrm{MeV}$. In this case no heavy fragments can be observed. What we call "clusters" in this work are different from heavy fragments in the previous sense. See Table I for a summary of the terminology in this paper. Clusters are the statistical correlation/association of $N$ nucleons appearing at the freeze-out stage of the collision. Its energy has a large uncertainty (proportional to $T_{f}$ ) and with overwhelming probability they decay into $N$ unbound nucleons at the post-freeze-out stage. Experimental evidence for cluster formation comes from the observed multiplicity distribution in certain detector acceptance (to be discussed below), or in production of $N=2,3$ nuclei $d$ and $t$.

Heavy-ion collisions at intermediate energies have been studied in the 1980s, both at CERN Super Proton Synchrotron and the Brookhaven National Laboratory Alternating Gradient Synchrotron, but not in sufficient detail. Many models predict that baryon-rich matter will also have the first-order transition line, ending in a certain critical point. Its search using enhanced fluctuations was proposed in Refs. [3,4]. The beam energy scan (BES) towards the lowest energies possible at RHIC is currently under way. Significant modification of the baryon number distributions, such as its large kurtosis, is indeed observed at the low energy end [5], perhaps indicating out-of equilibrium fluctuations related with criticality. Using the STAR detector at RHIC in a fixed-target mode is in the plans.

The topic of this paper is the baryon clustering phenomenon happening at the so-called freeze-out stages of heavy-ion collisions, in this intermediate baryon-rich domain. 
TABLE I. Terminology for different aggregations of nucleons used in this paper. We only address the formation of clusters (prenuclei) in this work.

\begin{tabular}{lccc}
\hline \hline Configuration & Temperature & Atomic number & Distribution \\
\hline Heavy fragments & $T<10 \mathrm{MeV}$ & $Z \gg 1$ & powerlike \\
Clusters (or prenuclei) & $T_{f} \sim 100-150 \mathrm{MeV}$ & $Z \gtrsim 1$ & Dispersion in energy \\
Light nuclei $\left(d, t,{ }^{4} \mathrm{He} \ldots\right)$ & observed at $T \ll T_{f}$ & $Z \gtrsim 1$ & (due to modified $N N$ potential) \\
thermal + nonequilibrium? & yes $\left(\Delta E \sim T_{f}\right)$ \\
\hline \hline
\end{tabular}

We will show how a relatively small modification of nuclear forces at distances $r=1-2 \mathrm{fm}$ can dramatically change the binding of baryonic clusters, as well as the kinetics of their production.

The paper is structured as follows. We start in Sec. II with the motivation for the modified internucleon potential, and present several versions of it-increasingly closer to the critical region-to be used in the following sections. To get some intuition on how these potentials affect the structure of nuclear matter, we present preliminary studies of how such modifications change the binding of clusters in Sec. III, using two opposite limits: the uncorrelated Gaussian-shape meanfield clusters, and fully correlated clusters forming classical shapes. Before describing our main body of simulations, we classify the observables to be used in Sec. IV. The bulk of our studies introduced in Sec. V is done with a classical dynamical approach, a molecular dynamics (MD) code complemented by Langevin (L) forces representing the effects of the mesonic heat bath. We proceed from a small number of nucleons and finite clusters in Sec. V, to rather large ones, with $N \sim 100$ particles in Sec. VI. In Sec. VII we present our main results in connection with the experimental conditions of the BES program at RHIC. On one hand we focus on the generated correlations among nucleons, and calculate the proton cumulants, as well as the scaled skewness and kurtosis of the distribution. Then, we study how strong correlations lead to the clustering of nucleons to form "prenuclei," and extract the number of "pre- ${ }^{4} \mathrm{He}$ " in the same simulations. Finally, in Sec. VIII we summarize and present our conclusions.

The remainder of this Introduction contains a summary of the ideas motivating this work.

One important notion is the very high sensitivity of the dynamical clustering to the details of the internucleon effective potential. Since the time of Yukawa's suggestion, nuclear forces are traditionally described in terms of certain meson exchanges. Furthermore, as all nuclear physicists know, any model of nuclear forces needs special tuning, needed to reproduce two delicate phenomena: (i) strong cancellation between repulsion and attraction in the mean potential energy; and (ii) partial cancellation of the remainder in the mean potential energy by quantum kinetic energy. The final result should be that neutron systems, and in fact many species of light nuclei, are not bound. Even infinite nuclear matter, with an equal number of protons and neutrons (and Coulomb effects switched off) is only slightly bound.

Because of these cancellations, a small modification of the internucleon potential can induce quite significant changes in binding, even up to an order of magnitude. This is of crucial importance, because the temperatures of the hadronic phase we discuss range from the critical temperature $T_{c} \approx$ $120-155 \mathrm{MeV}$ down to the kinetic freeze-out temperature of baryons $T_{\text {kin }} \approx 80-100 \mathrm{MeV}$. Such temperatures may appear large compared to the usual nuclear potential depth $\sim 50 \mathrm{MeV}$ and binding per nucleon $\sim 10 \mathrm{MeV}$. And yet, even with such conditions we do find significant clusters of trapped baryons. We therefore suggest to look not only at higherorder moments of the net-baryon distribution, but also outof-equilibrium production of light nuclei.

Why do we think that internucleon effective potentials might be modified in the conditions discussed, from wellknown forces in cold nuclear matter?

One generic reason-suggested many times before-is that in the baryon-rich end of the phase diagram certain modification of meson masses and couplings should be much larger than in the (well studied) small- $\mu_{B}$ meson-dominated regime. In the spirit of the resonance gas model, one may argue that there are many more baryonic resonances than mesonic ones. Studies of dilepton spectral density [6] and related $\rho$-meson modifications [7] have indeed shown such baryonic dominance. It is furthermore quite reasonable to think that what happens with $\rho$ should happen with other wide resonances, the $\sigma$ in particular.

Another generic reason, emphasized in Ref. [3] and also widely known, is the possible existence of the (hypothetical) $Q C D$ critical point, as the endpoint of the first-order phase transition line. On general theoretical grounds we know that second-order phase transitions have massless modes, which lead to the phenomenon of critical opalescence at scales much larger than the microscopic scales of matter. If exchanges of such long-range critical modes do appear in the internucleon potential — even with relatively small coupling — we will find a significant enhancement of both the binding of certain nuclear clusters, and the kinetic clustering rates.

Finally, as multiple studies on the kinetics near the phase transitions indicate, the so-called "critical slowing down" phenomenon prevents complete equilibration, and opens the door to multiple out-of-equilibrium scenarios, some with significant cluster production.

\section{FREEZE-OUT CONDITIONS AND MODIFIED BARYON POTENTIALS}

We already mentioned the resonance gas model, which is very successful for predicting hadronic yields for high-energy heavy-ion collisions. It is based on the standard statistical expression for the equilibrium particle densities at number of 
baryons of type $i$,

$N_{i}=\gamma_{i} V_{\mathrm{tot}} \int \frac{d^{3} p}{(2 \pi)^{3}} \frac{1}{\exp \left[\left(m_{i}-\bar{\mu}+p^{2} / 2 m_{i}\right) / T_{\mathrm{ch}}\right]+1}$,

where $\gamma_{i}, V_{\text {tot }}, T_{\mathrm{ch}}$ are the statistical weight, total effective volume of the chemical freeze-out surface, and the corresponding chemical freeze-out temperature. We put a bar on the chemical potential indicating that we include the mean value of the interbaryon potential in it, $\bar{\mu}=\mu-\bar{V}$.

There are certain important distinctions between highenergy collisions and the conditions we are going to study. First of all, in the former case $\mu_{B} \approx 0$ and baryons/antibaryons are both very much suppressed by the Boltzmann factor, since $m_{i} / T_{\text {ch }} \sim 10$. Second, at $T_{\text {ch }} \approx T_{c} \approx$ $155 \mathrm{MeV}$, excitation of baryonic resonances $N^{*}, \Delta^{*}$ and their strange counterparts is very significant. For example, the population of the $S=3 / 2, I=3 / 2 \Delta$ resonance, relative to that of the nucleon, is about $4 \exp \left[\left(m_{\Delta}-m_{N}\right) / T_{\mathrm{ch}}\right] \approx 0.7$. On the other hand, in the time between the chemical and kinetic freeze-out, with $T_{\text {kin }} \approx 80-100 \mathrm{MeV}$, most of them decay into a baryon and one (or more) mesons, providing large "feed-down corrections" to nucleon yields.

For the conditions of the BES, on the other hand, the chemical potential is in the range $\bar{\mu}=500-700 \mathrm{MeV}$, and the Boltzmann factors exp $\left[-\left(m_{i}-\bar{\mu}\right) / T_{\mathrm{ch}}\right]$ are not so punishingly small. Furthermore, the number of antibaryons is negligible, and we will not discuss them in the following. The chemical freeze-out temperature is lower, and thus a fraction of excited baryonic resonances is much smaller. In the following we will (maybe crudely) ignore their existence and feeddown. We thus normalize our calculations to the total final nucleon number observed experimentally. Another way to explain this approach is to realize that in the Serot-Walecka model we will use, the baryonic states have the same effective potentials as the nucleons. This is so because the effective Lagrangian does not depend on isospin. Then, the time evolution of any baryon follows the same MD $+\mathrm{L}$ equation independently of its nature as long as an average baryon mass is employed in the calculation.

Let us finally comment on the distinctions between our molecular dynamics computations and those for low-energy heavy-ion collisions. If the temperature $T \sim 10 \mathrm{MeV}$, the thermal kinetic energy is comparable with the Fermi energy of matter at nuclear densities, and therefore quantum effects play a significant role and need to be taken care of, by some kind of approximation. The freeze-out temperatures we deal with are significantly higher; many states are excited and the role of Fermi repulsion is significantly reduced.

Nucleons under freeze-out conditions populate a nondegenerate classical gas. In this case the number density per internal degree of freedom $n_{\mathrm{DOF}}$ should be small compared to the "quantum density,"

$$
n_{\mathrm{qu}}=\left(\frac{m T}{2 \pi \hbar^{2}}\right)^{3 / 2}
$$

Inserting the nucleon mass and temperatures in question, one finds that $n_{\mathrm{qu}}(T=150 \mathrm{MeV})=0.44 \mathrm{fm}^{-3}$. Nucleons have $(2 S+1)(2 I+1)=4$ internal degrees of freedom, so at nuclear matter density $n_{\mathrm{DOF}}=n_{0} / 4=0.04 \mathrm{fm}^{-3}$, which is indeed smaller than the $n_{\text {qu }}$ value above. Note that when the temperature is an order of magnitude smaller-as is the case in lower collision energies related to multifragmentation studies $-n_{\text {qu }}$ is reduced by a factor 30 , and the regime is reversed, $n_{\mathrm{qu}}<n_{\mathrm{DOF}}$, and matter is thus a degenerate Fermi gas. Nevertheless, we will study some effective quantum corrections in Appendix E.

Let us now proceed to the discussion of in-matter forces between the baryons, starting with the so called "mass shifts" issue, which is somewhat controversial. On one hand, a significant part of the nucleon mass is believed to be due to "constituent quark masses" induced by chiral symmetry breaking. If so, in view that the freeze-out is not far from the restoration line of the chiral symmetry, it was predicted by many phenomenological and theoretical models that there should be a significant downward shift of such contributions to the effective quark mass. On the other hand, as we mentioned already, the successful thermodynamical description of the particle yields at chemical freeze-out uses the resonance gas model without any modifications of the particle masses.

Furthermore, the range of the internucleon potentials is defined by masses of the corresponding mesons. For one of them, the vector meson $\rho$, we have direct access to its spectral density via the dilepton production, and its significant widening has indeed been observed [6]. For the $\omega$ meson no changes are observed, which is expected, since due to its longer lifetime most of them decay outside of the fireball. The $\sigma$ meson, wide even in vacuum [8], is often represented as a correlated $\pi \pi$ pair, and is perhaps getting even wider in matter. The effective potential, convoluting Yukawa potential with its spectral density (cf. Appendix B), is expected to become longer range or even infinite range at the critical point.

Unfortunately, lattice QCD at the moment can only extrapolate to $\mu_{B} / T<2$ or so, which is far from the regime we are interested in. Some hints can perhaps be gained from the lattice study by the Graz group [9], which performed restoration of chiral symmetry "by surgery" i.e., simply removing the lowest Dirac eigenstates from the hadronic mass evaluation. What is observed is that the chiral partners (such as the nucleon $P=+1$ and $N^{*} P=-1, \rho$ and $a_{1}$, etc.) modify their masses in the opposite directions, meeting somewhere in between. Perhaps such effects cancel each other in the calculations of the total baryon and meson yields. If so, note that the chiral partner of the $\sigma$ is the pion. Moving towards it means reducing its mass, maybe to a half of it, or even all the way to zero (close to the second-order phase transition).

Completing the motivation, we now explain the reader the simplified form of nuclear forces we will be using. It follows from the popular relativistic model by Serot and Walecka [10]. One important simplifying characteristic is that it only includes the isoscalar mesons, scalar $\sigma$ and vector $\omega$, so there is no difference between the interaction of protons and neutrons. We will also ignore electromagnetism, as the clusters studied are not so large as to make it important. 


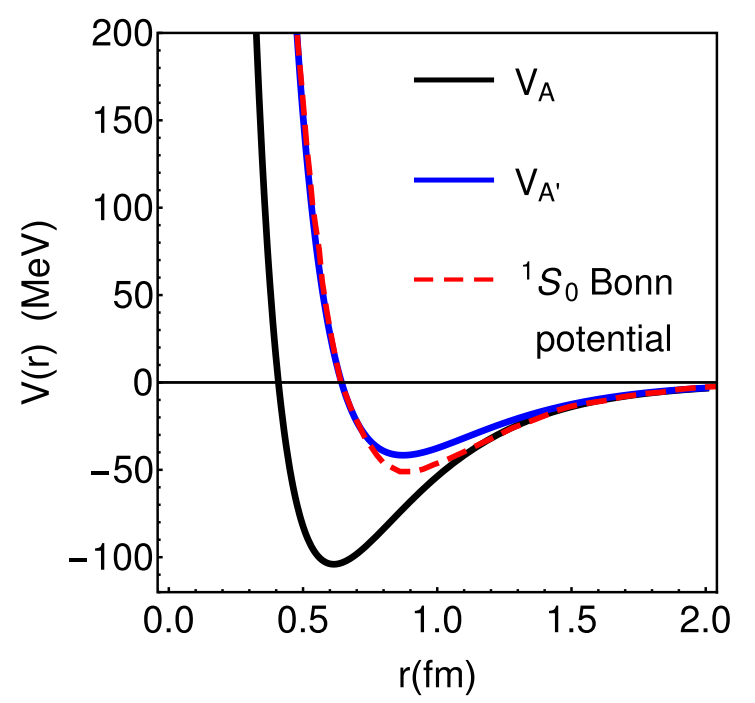

FIG. 1. Phenomenological nucleon-nucleon potentials. Solid line: Serot-Walecka potential (4) with parameters in (5). Dotted line: Same as before but with the repulsive strength increased by a factor 1.4. Dashed line: Bonn $N N$ potential [11] in ${ }^{1} S_{0}$ channel, taken from [12].

The Lagrangian density of their model is

$$
\begin{aligned}
\mathcal{L}= & \frac{1}{2}\left(\partial_{\mu} \phi \partial^{\mu} \phi-m_{\sigma}^{2} \phi^{2}\right)-\frac{1}{4} F_{\mu \nu} F^{\mu \nu}+\frac{1}{2} m_{\omega}^{2} V_{\mu} V^{\mu} \\
& +\bar{\psi}\left[\gamma_{\mu}\left(i \partial^{\mu}-g_{\omega} V^{\mu}\right)-\left(m_{N}-g_{\sigma} \phi\right)\right] \psi,
\end{aligned}
$$

where the Abelian field strength of the vector field $F_{\mu \nu} \equiv$ $\partial_{\mu} V_{\nu}-\partial_{\nu} V_{\mu}$ is the same as in electrodynamics. There are thus three fields, Dirac nucleons $\psi$, vector $\omega$ mesons $V_{\mu}$, and scalar $\sigma$ mesons $\phi$, interacting with each other in a relativistically invariant way. Their masses are considered to be an input. For definiteness we use $m_{\sigma}=500 \mathrm{MeV}, m_{\omega}=782 \mathrm{MeV}$, and $m_{N}=938 \mathrm{MeV}$.

The resulting static potential between nucleons is

$$
V_{A}(r)=-\frac{g_{\sigma}^{2}}{4 \pi r} e^{-m_{\sigma} r}+\frac{g_{\omega}^{2}}{4 \pi r} e^{-m_{\omega} r},
$$

where the coupling values selected by Serot and Walecka [10] are

$$
g_{\sigma}^{2}=267.1\left(\frac{m_{\sigma}^{2}}{m_{N}^{2}}\right), \quad g_{\omega}^{2}=195.9\left(\frac{m_{\omega}^{2}}{m_{N}^{2}}\right)
$$

The $\omega$ coupling is stronger, thus dominating at small distances. Note further that these two terms nearly cancel each other, leaving us with a relatively shallow potential, $\left|V_{A}\right|<$ $100 \mathrm{MeV} \sim m_{N} / 10$; see Figs. 1 and 25. It is also important to notice that the couplings are selected not to fit the binary scattering phase shifts and deuteron binding, as done for all other phenomenological potentials, but from the fit to nuclear matter in the mean-field approximation. The details of that are further delegated to Sec. III C.

For our studies of the baryonic clustering in this work we will use the Serot-Walecka model in four different versions of the mesonic masses:

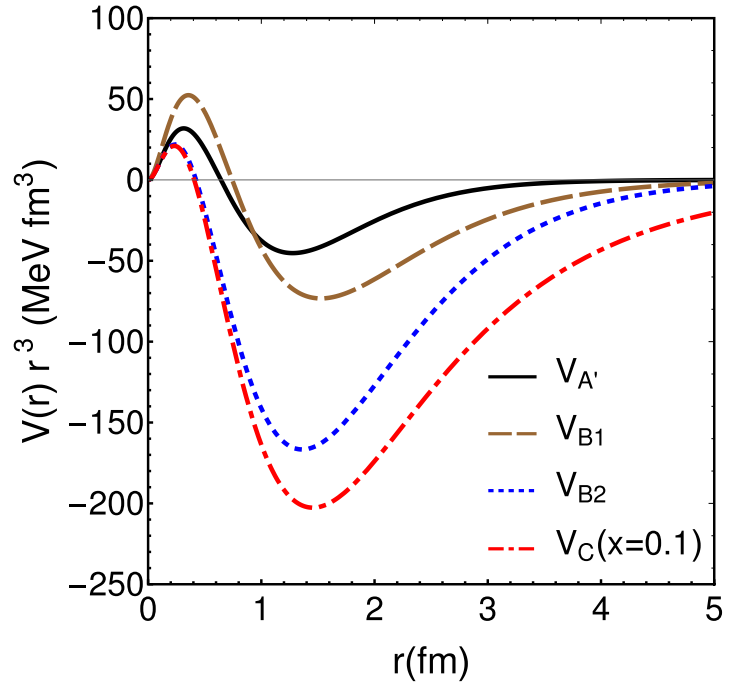

FIG. 2. $V(r) r^{3}\left(\mathrm{MeV} \mathrm{fm}^{3}\right)$ vs $r(\mathrm{fm})$ for the four models used in this work. The original Walecka potential with increased repulsion (potential $A^{\prime}$ ) is shown by the black solid line, the versions $B 1$ and $B 2$ correspond to the dashed brown and dotted blue dashed lines, respectively. The version $C$ potential, with $x=0.1$ is represented by the red dash-dotted line.

(A) The unmodified Walecka potential (4) with the parameters computed at mean field quoted in (5).

$\left(\mathrm{A}^{\prime}\right)$ Walecka potential with increased repulsion $g_{\omega}^{2} \rightarrow$ $1.4 g_{\omega}^{2}$ to make it closer to the phenomenological Bonn potential.

(B1) One in which the $\sigma$ mass squared decreases "halfway" (that is $m_{\sigma}^{2} \rightarrow m_{\sigma}^{2} / 2$ ), presumed to hold at the critical line for $\mu_{B}<\mu_{c}$. The "minimal modification" version changes the coupling as well, $g_{s}^{2} \rightarrow$ $g_{s}^{2} / 2$, keeping the mean potential energy constant.

(B2) This version is the same as (B1) except that the scalar coupling is not modified. The mean potential from $\sigma$ thus is a factor 2 larger than in $(B 1)$.

(C) An admixture of the (B2) potential with the one with very light critical mode $\sigma, m_{\sigma}^{2} \rightarrow m_{\sigma}^{2} / 6$ (denoted as $V_{\text {crit }}$,

$$
V_{C}(r ; x)=(1-x) V_{B 2}(r)+x V_{\text {crit }}(r) .
$$

In Fig. 2 we show the corresponding potentials, multiplied for convenience by $r^{3}$ (note that $4 \pi r^{3} / 3$ times the density of other baryons tell us effectively how many "partners" a given baryon has). As one can see, these four models show progressively increasing depth and range of the attractive potential.

\section{PRELIMINARY STUDIES OF CLUSTER BINDING}

Before we discuss our dynamical out-of-equilibrium studies of multibaryon systems, it is instructive to report some simplified approaches. We considered either $N=4-13 \mathrm{nu}-$ cleons, or clusters of certain fixed size, and use all versions of the modified potentials described above. In Sec. III A we consider a limit in which there are no correlations between locations of the nucleons, so that the $N$-body distribution is 
simply factorizable into a product

$$
n\left(\vec{r}_{1}, \vec{r}_{2}, \ldots, \vec{r}_{N}\right)=\prod_{i} n\left(\vec{r}_{i}\right),
$$

with the same Gaussian-like spatial distribution. In Sec. III B we turn to the opposite limit, in which the nucleons are set to specific locations, defined by symmetry considerations, which in turn depend on the particle number, and study the dependence of the total energy on the scale parameter. Finally, in Sec. III C we calculate properties of self-consistent meanfield clusters, formed of only bound nucleons.

\section{A. Clusters made of uncorrelated nucleons}

In this section we illustrate the effect of the different potentials defined above in a simple mean-field (no correlations) model. Let us consider a Gaussian-shaped cluster, with the nuclear matter density $n_{0}=0.16 \mathrm{fm}^{-3}$ at its core,

$$
n(r)=n_{0} \exp \left(-\frac{r^{2}}{2 R^{2}}\right)
$$

and the r.m.s. size $R=2 \mathrm{fm}$. The integral $N=\int d^{3} r n(r) \approx$ 20 , so this is a crude model of a medium-size nucleus.

Using the Thomas-Fermi expression for local Fermi momentum with $\gamma$ degrees of freedom $p_{F}(r)=\left[6 \pi^{2} n(r) / \gamma\right]^{1 / 3}$ one can calculate the kinetic energy per nucleon. For $\gamma=4$ it is

$$
\frac{K}{N}=\frac{1}{N} \int d^{3} r \frac{3}{5} \frac{p_{F}(r)^{2}}{2 m_{N}} n(r) \approx 10.2 \mathrm{MeV},
$$

independent or $R$. For pure neutron matter with $\gamma=2$ we get $K / N \approx 16.2 \mathrm{MeV}$.

Now, ignoring pair correlations $n\left(\vec{r}_{1}, \vec{r}_{2}\right) \rightarrow n\left(\vec{r}_{1}\right) n\left(\vec{r}_{2}\right)$ (mean-field approximation), one can calculate the potential energy

$$
P=\frac{1}{2} \int d^{3} r_{1} d^{3} r_{2} n\left(r_{1}\right) V\left(\vec{r}_{1}-\vec{r}_{2}\right) n\left(r_{2}\right),
$$

corresponding to forces defined in the preceding section. The results are $P=-15.1,3.7,6.7,-52.5$, and $-70.9 \mathrm{MeV}$ per nucleon, for models $A, A^{\prime}, B 1, B 2$, and $C$, respectively. In Fig. 3 we summarize our results for the total energy per nucleon, for the different potentials used in this work. We also consider different r.m.s. of the nuclear density, with a total number of nucleons of 2.5 (circles), 8.5 (squares), and 20 (triangles).

The total energy per nucleon $(K+P) / N$ for the Walecka model $A$ (whose parameters are chosen at mean field) is $\sim-5 \mathrm{MeV}$ for nuclear matter ( $p, n$ equal mixture), and $+1 \mathrm{MeV}$ for pure neutrons.

The model $A^{\prime}$ is chosen to be much more shallow than the previous Walecka potential, and it is not able to bind this kind of clusters in the mean-field approximation. Similarly, the potential $B_{1}$ has a comparable effect, with a total energy per nucleon of several dozens of MeV. However models $B 2$ and $C$ lead to a large binding. As we will detail later, the addition of binary correlation function can increase this binding even more. The main lesson from this initial calculation is that significant binding (non-negligible compared to temperatures

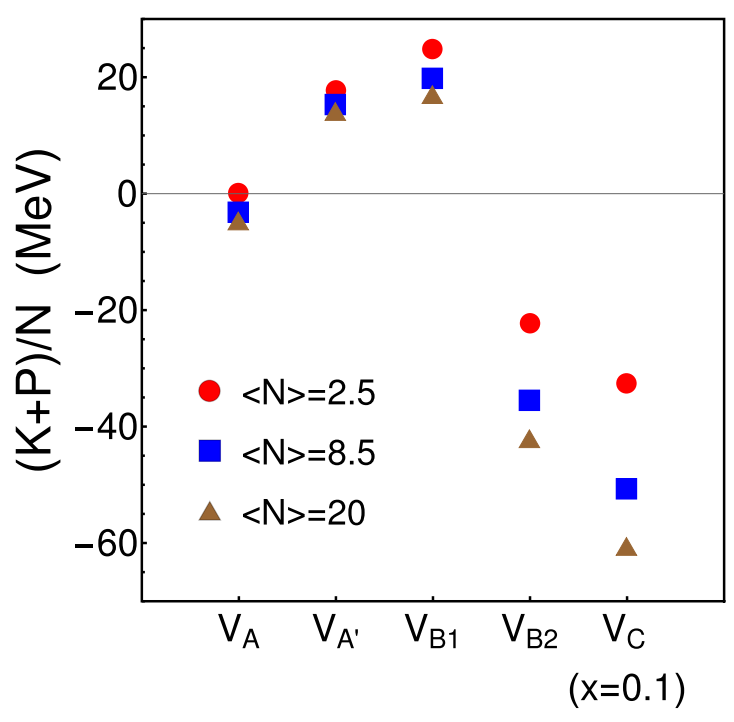

FIG. 3. The total energy per nucleon in $\mathrm{MeV} /$ nucleon, for all versions of the binary potentials and the number of nucleons $N=2.5,8.5,20$; corresponding to Gaussian density with r.m.s. radii $R=1,1.5,2 \mathrm{fm}$, respectively.

of $T \approx 100 \mathrm{MeV}$ ) can be produced at mean field only for significantly modified potentials (models $B 2$ and $C$ ). We study now the effect of correlations in such systems.

\section{B. Clusters made of strongly correlated nucleons}

For vanishingly small temperatures and small values of the particle number $N$, the geometry of the classical lowest energy states is suggested by symmetry. In this section we present some expectations as functions of $N$. Later, we will study not only the near-freeze-out $T \sim 100 \mathrm{MeV}$ cases, but also cool the systems down to $T \approx 1 \mathrm{MeV}$ and even $T \approx 10^{-3} \mathrm{MeV}$ and test that the symmetric configurations considered in this section are indeed obtained from the MD $+\mathrm{L}$ simulations.

For definiteness, the potential used in this section is $V(r)=$ $V_{A^{\prime}}(r)$, which is enough to bind the nucleons when no thermal effects are accounted for $(T=0)$.

The smallest number of particles we consider is $4, N=4$, which form a tetrahedron. As it is known from studies of fewbody nuclei, such correlation between four nucleons is indeed rather strong inside the ${ }^{4} \mathrm{He}$, and persists in "alpha-particle nuclei" such as ${ }^{12} \mathrm{C},{ }^{16} \mathrm{O}$. All six pair distances between the four nucleons are in this case the same, denoted by $a$. For a general $N, a$ is defined as the minimum distance between 2 nucleons in equilibrium - which is not necessarily the minimum of the internucleon potential. The energy per nucleon $\langle V\rangle_{N}$ in this simplest case is just

$$
\langle V\rangle_{4}=\frac{3}{2} V(a)
$$

The octahedron has $N=6$ particles and 15 pairs: 12 of them of distance $a$ and 3 of distance $\sqrt{2} a$. The energy per nucleon is in this case

$$
\langle V\rangle_{6}=2 V(a)+\frac{1}{2} V(\sqrt{2} a) .
$$




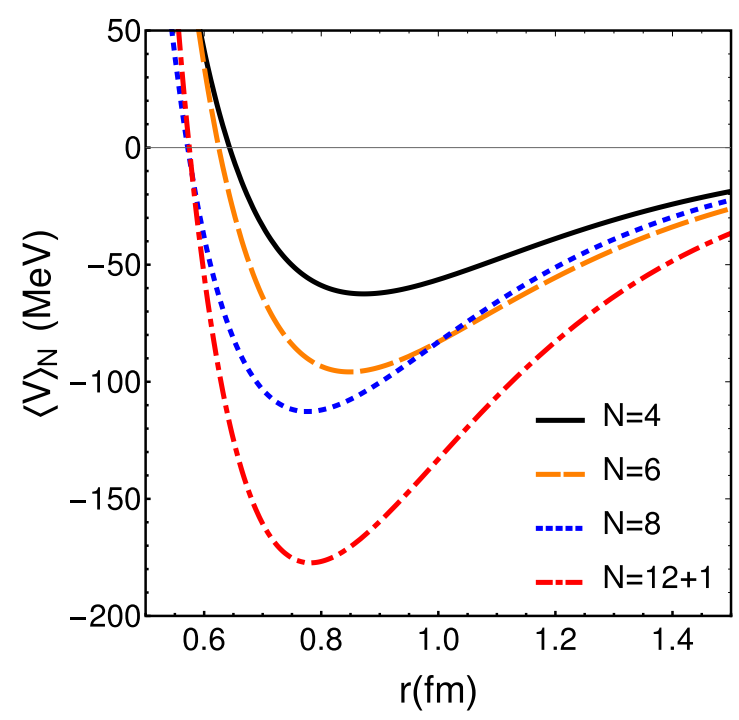

FIG. 4. The potential energy per particle $\langle V\rangle_{N}$ (MeV/nucleon) as a function of distance $r(\mathrm{fm})$ for four clusters: $N=4$ tetrahedron (the upper thin solid line), $N=6$ octahedron (the dashed line), $N=8$ cube (the dotted line), and $N=12+1$ icosahedron + one particle (the lower dot-dashed line). This calculation is done with the Walecka potential $V_{A^{\prime}}$.

The next cluster to consider, of $N=8$ particles, is the hexahedron (or cube). It has 12 distances $a, 12$ distances $\sqrt{2} a$, and four distances $\sqrt{3} a, 28$ in total,

$$
\langle V\rangle_{8 h}=\frac{3}{2} V(a)+\frac{3}{2} V(\sqrt{2} a)+\frac{1}{2} V(\sqrt{3} a) .
$$

The largest particular cluster we discuss is the icosahedron with 12 vertices, to which we added one particle at the center, making $N=13$. It has 78 distances: 12 distances at $a, 30$ distances at $\sqrt{2-2 / \sqrt{5}} a, 30$ at $\sqrt{2+2 / \sqrt{5}} a$, and 6 at $2 a$,

$$
\begin{aligned}
\langle V\rangle_{12+1}= & \frac{12}{13} V(a)+\frac{30}{13} V(\sqrt{2-2 / \sqrt{5}} a) \\
& +\frac{30}{13} V(\sqrt{2+2 / \sqrt{5}} a)+\frac{6}{13} V(2 a) .
\end{aligned}
$$

The energy per particle $\langle V\rangle_{N}(r)$ for all four clusters, as a function of the distance $r$, is shown in Fig. 4. One can see that augmenting $N$ this potential energy increases, eventually exceeding the range of temperatures in the problem $T=$ (100-150) MeV by a significant factor (even assuming that the potential is not modified by the temperature). Previous experience of working with strongly coupled Coulomb plasmas, see Ref. [13] and references therein, tells us that for such range of $\langle V\rangle_{N} / T$ the factorized mean-field theory is completely inadequate, and the correlations are significant. At the same time, this range of the ratio is also too small to cause solidification of the system, keeping the system in the strongly correlated but still liquid phase.

The value of the minimal distance between two nucleons in equilibrium was denoted by $a$, and it can be obtained by minimizing the potential energy per particle in Fig. 4 for each $N$. For future reference, we summarize these distances and the corresponding potential energy in Table II. Notice that $a$
TABLE II. Minimal distance between nucleons and potential energy per nucleon for several configurations with $N$ nucleons.

\begin{tabular}{lllc}
\hline \hline$N$ & \multicolumn{1}{c}{ Polyhedron } & $a(\mathrm{fm})$ & $\langle V\rangle_{N}(\mathrm{MeV} /$ nucleon $)$ \\
\hline 4 & tetrahedron & 0.8727 & -62.47 \\
6 & octahedron & 0.8481 & -95.78 \\
$8 h$ & hexahedron (cube) & 0.7761 & -112.70 \\
$8 s$ & square antiprism & 0.8096 & -117.03 \\
$12+1$ & icosahedron +1 & 0.7816 & -177.32 \\
\hline \hline
\end{tabular}

coincides with the minimum of the potential $V_{A^{\prime}}(r)$ only for the case $N=4$.

Finally note that suggested by a totally different minimization problem (Thomson problem in electrostatics [14]), we have tried a different configuration for $N=8$, the square antiprism, whose energy per particle is denoted as $\langle V\rangle_{8 s}$. We indeed find a lower potential energy than the cubic configuration, providing an example where the expectation based on symmetry (coming in this case from the Platonic solids) does not provide the optimal configuration. We will come back to these geometries when applying our $\mathrm{MD}+\mathrm{L}$ simulations to cold systems.

\section{Mean-field baryon clusters at freeze-out}

Before we study the clustering phenomenon dynamically, it is important to see what kind of clusters can in principle be self-consistent, in analogy to globular clusters in galaxies.

Let us assume homogeneous matter at rest, with certain mean density (1) and the mean potential $\bar{V}$, and on top of it a cluster, as a deviation from the mean. It is cause by a deviation of the mean potential $\delta V(r)=V(r)-\bar{V}$. In thermal equilibrium it will add an extra density of baryons,

$$
\begin{aligned}
\delta n_{i}(r)= & \gamma_{i} \int \frac{d^{3} p}{(2 \pi)^{3}}\left[\frac{1}{\exp \left(\frac{m_{i}-\bar{\mu}+\frac{p^{2}}{2 m}+\delta V(r)}{T_{\mathrm{ch}}}\right)+1}\right. \\
& \left.-\frac{1}{\exp \left(\frac{m_{i}-\bar{\mu}+\frac{p^{2}}{2 m}}{T_{\mathrm{ch}}}\right)+1}\right] .
\end{aligned}
$$

Furthermore, following the setting of the globular clusters in the galaxies described in Appendix D, we will consider times at which all unbound particles have already left the cluster, and in the phase space integral we include only bound particles. This means in the momentum integral we only integrate over the region where

$$
\frac{p^{2}}{2 m}+\delta V(r)<0
$$

To make a cluster self-consistent, this extra potential $\delta V(x)$ should be created by the extra density itself. We write this condition in the integral form

$$
\delta V\left(\vec{r}_{1}\right)=\int d^{3} r_{2} V\left(\vec{r}_{1}-\vec{r}_{2}\right) \delta n_{i}\left(\vec{r}_{2}\right),
$$


TABLE III. The parameters of the self-consistent clusters for various input potentials, all with the same r.m.s. radius $R=2.2 \mathrm{fm}$. $N$ is the integrated number of baryons in the cluster, $\langle V\rangle / N$ and $V(r=0)$ are the mean potential per baryon, and the potential depth at the center.

\begin{tabular}{lrcc}
\hline \hline Potential & $N$ & $\langle V\rangle / N(\mathrm{MeV})$ & $V(r=0)(\mathrm{MeV})$ \\
\hline$A$ & 25.4 & -180 & -295 \\
$B 2$ & 10.5 & -113 & -207 \\
$C$ & 7.8 & -119 & -187 \\
\hline \hline
\end{tabular}

equivalent to the Poisson Eq. (D8) for the Newtonian potential in Appendix D. The two equations (15) and (17) together make a system of equations which needs to be solved.

One simplification is to ignore +1 in (15), that is to proceed from Fermi to Boltzmann statistics. Note further that when $\delta V / T$ is small, one can expand the bracket to the first order in it, and then take the momentum integral using the binding condition (16). The resulting contribution is $\delta n \sim \delta V^{5 / 2}$. The exact integral without expansion can also be done analytically, leading to the following function of $z \equiv \delta V / T$, given with its (rather well convergent) series:

$$
\begin{aligned}
N(z)= & e^{z} \operatorname{Erf}(\sqrt{z})-\frac{2 \sqrt{z}(3+2 z)}{3 \sqrt{\pi}} \\
& +\frac{8 z^{5 / 2}}{15 \sqrt{\pi}}\left(1+\frac{2 z}{7}+\frac{4 z^{2}}{63}+\frac{8 z^{3}}{693}+\frac{16 z^{4}}{9009}+\cdots\right)
\end{aligned}
$$

[see also Eq. (D7)].

In practice we adopt the following procedure: start with a certain ansatz for $\delta V(r)$, e.g., Gaussian with two parameters, the amplitude and the radius. Then, via the $N(z)$ function, calculate numerically the right-hand side of Eq. (17), and tune the parameters to minimize the difference between the left-hand side, the obtained $\delta V$, and the input one. Of course, inside a given variational ansatz one cannot get a very good match of the shape, but the overall difference was kept at a reasonable level, of the order of $15-20 \%$.

We found it instructive to keep the radius of the cluster fixed, say r.m.s. radius $R=2.2 \mathrm{fm}$, and modify only the potential depth. For different potentials defined above, we find the best depth of the potential: the resulting number of nucleons in the cluster and the mean potential per nucleon in it; see Table III. One can see that while the original Walecka potential requires a quite deep potential and a large number of baryons, the modified potentials $B 2, C$ expected near the critical point can, due to its longer range, bind a smaller number of nucleons.

\section{OBSERVABLES}

In this section we include some generic discussion of the observables involved.

The thermodynamical susceptibilities in equilibriumderivatives of $\log Z$ over various chemical potentials of three light quarks—are usually recombined into

$$
c\left(N_{B}, N_{Q}, N_{S}\right)=\frac{\partial^{N_{B}+N_{Q}+N_{S}}}{\partial^{N_{B}} \mu_{B} \partial^{N_{Q}} \mu_{Q} \partial^{N_{S}} \mu_{S}}(\log Z) .
$$

We would call those global observables, because they correspond to mean correlation functions of fully integrated quark densities. Many of these quantities, up to $N=N_{B}+N_{Q}+$ $N_{s}=6$, are currently calculated on the lattice; see Ref. [15]. For their comparison to the heavy-ion data on event-by-event fluctuations see, e.g., Ref. [16].

At the opposite end are what we will call local observables, related to unintegrated local densities, for example, bilocal distribution function $n\left(\vec{r}_{1}, \vec{r}_{2}\right)$, which is usually defined in a "uncorrelated plus a correlation" form. In homogeneous matter it is defined as

$$
\left\langle n\left(\vec{r}_{1}, \vec{r}_{2}\right)\right\rangle=\left\langle n\left(\vec{r}_{1}\right)\right\rangle\left\langle n\left(\vec{r}_{2}\right)\right\rangle+C_{2}\left(\vec{r}_{1}-\vec{r}_{2}\right) .
$$

Similar definitions can be given for the $\mathrm{N}$-point correlators. Obviously, the local observables include the full information about the correlations in the system. However, for $N>2$ they are multidimensional functions, which is difficult to work with. Say, for $N=4$ and homogeneous matter, there are six relative distances, and it is not practical to calculate six-dimensional histograms.

Furthermore, as we will see, in bound clusters there are strong velocity-position correlations, so that in the classical approaches we adopt below one has to work with the phase space distributions, e.g. six-dimensional one body distribution $f(\vec{r}, \vec{v})$. Their local-in-phase-space correlators obviously are even of higher dimensions.

As a result, one needs to invent/use certain observables in between global and local ones. Experimentalists naturally use what we would call semiglobal observables, in which an integral is done over the detector acceptance. For example, it can be a certain range of longitudinal rapidities $y \in[-Y, Y]$ and transverse momenta $p_{\perp} \in\left[p_{\perp, \min }, p_{\perp, \max }\right]$. Typically, the included kinematic range is comparable to the excluded one, colloquially known as 50-50 percent setting, maximizing the fluctuations. One can measure distributions in the number of net protons $P\left(N_{p}\right)$, or electric net charge $P(Q)$, or net strangeness $P(S)$, deduce the corresponding moments, cumulants, etc., or correlations between these charges.

As will be discussed later, for the net-proton case, the kinematical cuts imposed in experimental analyses reduce the measured multiplicities by a factor around $5-15 \%$ of the total multiplicity (not really following 50-50 setting). Such a reduced multiplicity allows us to reach Poissonian fluctuations of protons and antiprotons, thus observing, for high energies, the Skellam expectations.

Another natural set of observables, which we would call semilocal ones, are those in which the densities (in coordinate space or the phase space) are integrated, but over the same small volume $V$,

$$
C(V, N)=\int_{V} \prod_{i}^{N} d^{3} r_{i}\left\langle n\left(\vec{r}_{1}\right) \ldots n\left(\vec{r}_{N}\right)\right\rangle
$$


(or analogous small region in the phase space). In studies of clustering we will do, the effect is of course maximal when $V$ is of the order of the volume of the clusters produced.

The last set of observables can in fact be directly observed in experiment, via physical clusters in the final state. One well known indicator of the baryon clustering is the deuterium $d$ production. The so-called coalescence models assume that $d$ yield is proportional to

$\int d^{3} r_{1} d^{3} r_{2} d^{3} p_{1} d^{3} p_{2} W_{d}\left(\vec{r}_{1}-\vec{r}_{2}, \vec{p}_{1}-\vec{p}_{2}\right)\left\langle f\left(\vec{r}_{1}, \vec{r}_{2}, \vec{p}_{1}, \vec{p}_{2}\right)\right\rangle$,

where $W_{d}$ is the so-called Wigner function related to the deuteron wave function. In this case the microscopic volume $V$ is that of the deuterons or other light nuclei, such as $t,{ }^{3} \mathrm{He},{ }^{4} \mathrm{He}$ (and hypernuclei, and their antiparticles), currently observed.

With out-of-equilibrium production of ${ }^{4} \mathrm{He}$ in mind, we will use below a four-particle observable, a normalized sum of six interparticle distances.

\section{MODEL: MOLECULAR DYNAMICS + LANGEVIN SIMULATIONS}

For the rest of the results in this paper we will use molecular dynamics (MD) simulations to study the agglomeration of nucleons and the time scales required for cluster formation. Previous applications of MD in nuclear matter to study clustering can be seen in Refs. $[17,18]$. We start by testing our code checking total energy and momentum conservation for finite systems, and then proceed to relatively large number of particles. These are contained in a cubic box with periodic boundary conditions and "reflections" on all sides, simulating infinite homogeneous matter. We reproduced a number of correlation functions for gas, liquid, and solid argon in a comparable regime; see Appendix C. We also apply the same approach to a (modified) Walecka potential to access the average properties of cold nuclear matter, introducing an effective quantum localization potential; see Appendix E. We relegate that study to an Appendix because it turns out not to be important for systems at temperatures around the freeze-out one.

Furthermore, we modify the MD code for a nuclear system at fixed temperature, using Langevin dynamics. The corresponding stochastic forces can be thought of as interactions of ambient heat bath made of multiple mesons (pions, kaons, etc.).

Presenting the results, we begin with systems with a small number of nucleons, starting with $N=4$. Using different temperatures, we check that they group into an average tetrahedral shape minimizing their energy per nucleon by sitting at mutual distances close to the minimum of the potential. Then, we will consider a larger number of nucleons and analyze their clustering rate. We will study the nuclear density profile of these clusters and their higher-order correlation functions.

\section{A. Setting}

A system with a small number of nucleons is useful to check and validate our $\mathrm{MD}+\mathrm{L}$ code. Equilibrium configu- rations can be easily found for such systems. In finite systems we find no extra complications due to periodic boundary conditions, such as breaking of the periodicity of the pairwise potential. Nevertheless, to avoid the particles escaping from the region of interest we sometimes implement a confining potential,

$$
U_{c}(|\vec{x}|)=V_{W S}(|\vec{x}|)-V_{W S}(0),
$$

which is written in terms of the Woods-Saxon potential,

$$
V_{W S}(|\vec{x}|)=-\frac{V_{0}}{1+\exp \left(\frac{|\vec{x}|-R}{a}\right)},
$$

where $V_{0}$ is the strength of the potential, $R$ is the radius of the volume, and $a$ is the skin depth. Such potential does not appreciably modify the dynamics in the region $|\vec{x}| \simeq 0$.

The temperature of the system is fixed by the light degrees of freedom (pions and kaons), which we encode in the nucleon Langevin dynamics. Therefore, we introduce a stochastic force to the nucleons as well as a drag force proportional and opposed to the nucleon momentum,

$$
\begin{aligned}
& \frac{d \vec{x}_{i}}{d t}=\frac{\vec{p}_{i}}{m_{N}}, \\
& \frac{d \vec{p}_{i}}{d t}=-\vec{\nabla} U_{c}\left(\left|x_{i}\right|\right)-\sum_{j \neq i} \frac{\partial V\left(\left|\vec{x}_{i}-\vec{x}_{j}\right|\right)}{\partial \vec{x}_{i}}-\lambda \vec{p}_{i}+\vec{\xi}_{i},
\end{aligned}
$$

where $\lambda$ is the drag coefficient and $\vec{\xi}$ is the random noise following a white Gaussian distribution,

$$
\begin{aligned}
\left\langle\vec{\xi}_{i}(t)\right\rangle & =0, \\
\left\langle\xi_{i}^{a}(t) \xi_{j}^{b}\left(t^{\prime}\right)\right\rangle & =2 T \lambda m_{N} \delta^{a b} \delta_{i j} \delta\left(t-t^{\prime}\right),
\end{aligned}
$$

with $a, b=1,2,3$ and we made use of the fluctuationdissipation theorem to relate the drag coefficient with the variance of the fluctuation noise. A reasonable value for $\lambda$ is taken from the baryon diffusion coefficient

$$
\lambda=\frac{T}{m_{N} D_{B}},
$$

where the latter is extracted from URASiMA simulations for similar conditions of density and temperature as those used here for the hadronic evolution until freeze-out [19], which is found to be around $D_{B} \simeq 0.5 \mathrm{fm}$. Incidentally this number is not too far from the often quoted estimate using strongly coupled QGP from holography [20] $D_{B} \simeq(2 \pi T)^{-1}$ for temperatures around $T_{\mathrm{ch}}=120 \mathrm{MeV}$.

The final value used in our simulations will be $\lambda=$ $0.256 \mathrm{fm}^{-1}$. The precise number is not important as long as it allows for a rapid thermalization of the system.

\section{B. Few-nucleon configurations}

It is instructive to remind the different distribution of distances for the first Platonic polyhedra discussed in Sec. III B. We summarize them in Table IV.

\section{1. $N=4:$ Tetrahedron}

We first apply this MD scheme to a system of $N=4$ particles and $V\left(x_{i j}\right)=V_{A}\left(x_{i j}\right)$, i.e., the unmodified Walecka 
TABLE IV. Summary of the distances of edges of some polyhedra. $a$ denotes the length of the minimal edge. Included for completeness the cube configuration does not appear as an equilibrium configuration, rather the square antiprism. We denote $\phi_{ \pm} \equiv \sqrt{2 \pm 2 / \sqrt{5}}$.

\begin{tabular}{llcc}
\hline \hline$N$ & \multicolumn{1}{c}{ Polyhedron } & Distances of edges & Proportion \\
\hline 4 & tetrahedron & $a$ & 6 \\
6 & octahedron & $a, a \sqrt{2}$ & $12: 3$ \\
$8 h$ & hexahedron (cube) & $a, a \sqrt{2}, a \sqrt{3}$ & $12: 12: 4$ \\
$8 s$ & square antiprism & $a, a \sqrt{2}, a \sqrt{1+\sqrt{2}}$ & $16: 4: 8$ \\
13 & icosahedron+1 & $a, a \phi_{-}, a \phi_{+}, 2 a$ & $12: 30: 30: 6$ \\
\hline \hline
\end{tabular}

potential. We first run a simulation at very low temperatures $T=10^{-3} \mathrm{MeV}$ to match the analysis in Sec. III B. The initial sampling of velocities is done at higher temperatures so that the particles are given some time to acquire their equilibrium configuration. The evolution of the potential, kinetic, and total energies is seen in the top panel of Fig. 5. While the kinetic energy is negligible, the potential energy per nucleon takes the equilibrium value $\langle V\rangle_{N}=-62.47 \mathrm{MeV}$ (as predicted from Table II).
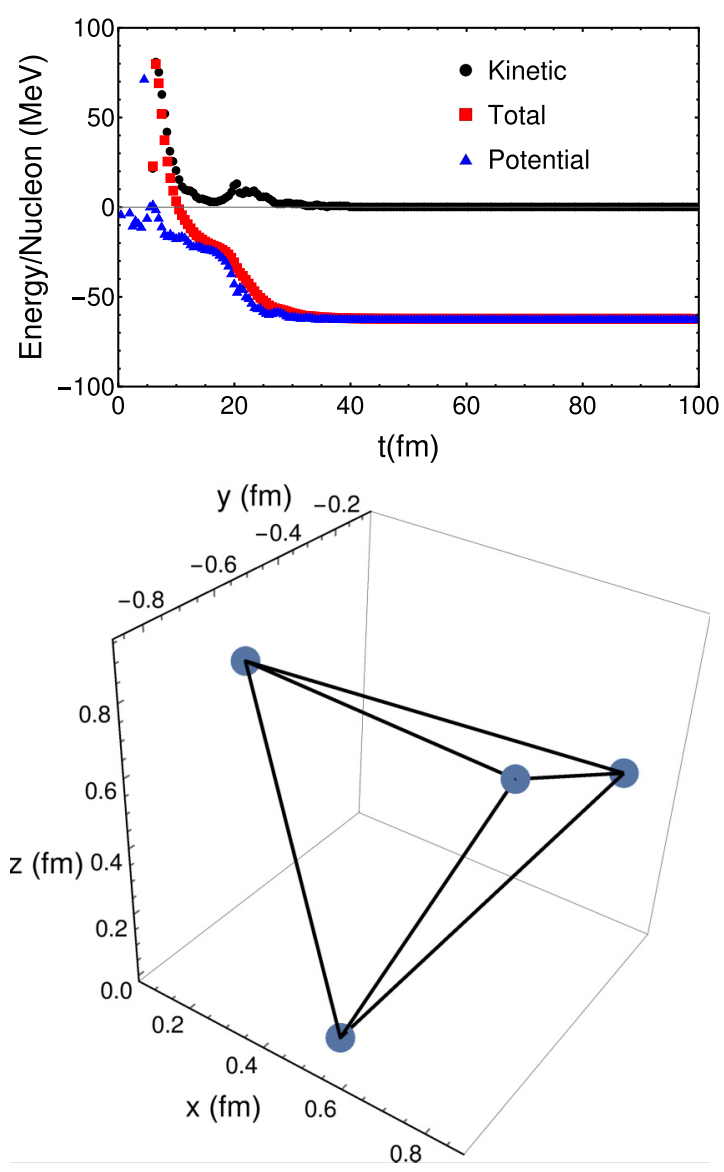

FIG. 5. Top: Kinetic (black), potential (blue), and total (red) energies per nucleon (in $\mathrm{MeV}$ ) for $N=4$ calculation vs time (fm) at $T=10^{-3} \mathrm{MeV}$. Bottom: Snapshot of the coordinate configuration at some time after equilibration.
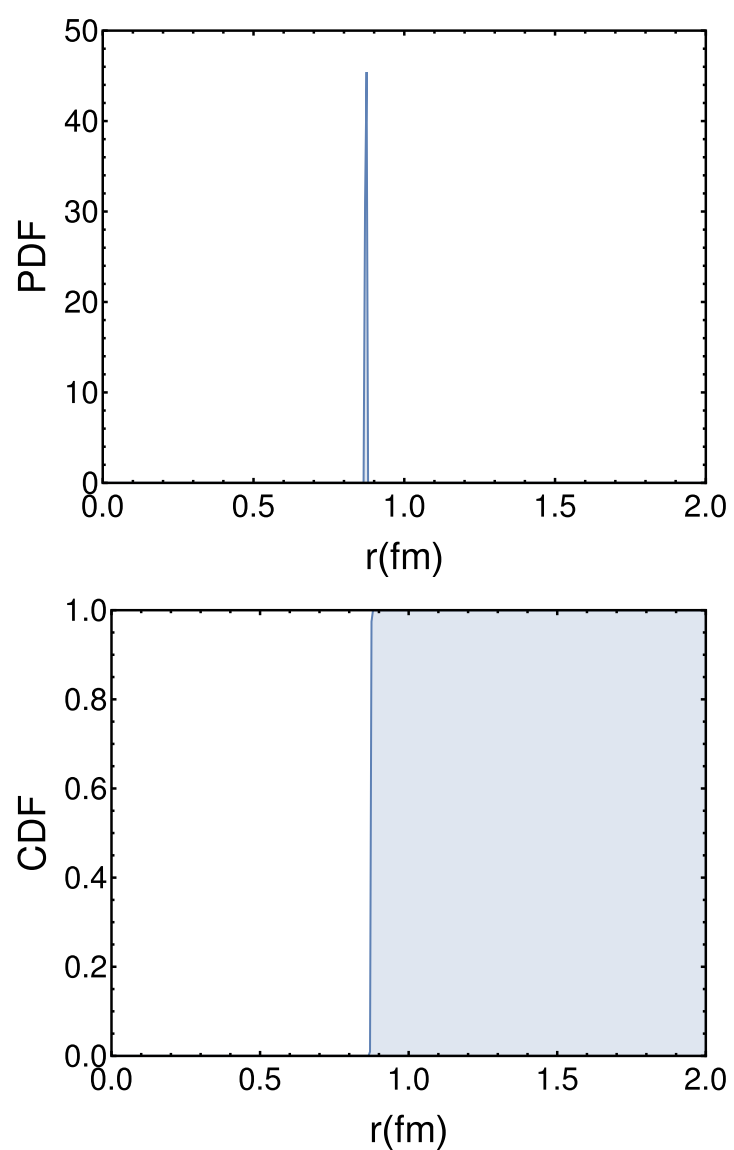

FIG. 6. Top: Histogram of the distance between nucleon pairs for $N=4$ simulation at $T=10^{-3} \mathrm{MeV}$. Bottom: Cumulative distribution function.

It is easy to see that the geometrical configuration is the expected tetrahedron shape (Fig. 5, bottom), whose center of mass is evolving with time but the relative distances are preserved. We perform a (time) distribution of the distances between pairs of nucleons in the top panel of Fig. 6. The probability distribution function (PDF) shows a single peak at $0.873 \mathrm{fm}$, which is the expected value quoted in Table II, and corresponds to the minimum of the Walecka potential $V_{A}$. The cumulative distribution function (CDF) jumps from 0 to 1 precisely at this distance (bottom panel of the same figure). These distribution functions-not particularly informative in this case-will become useful for the cases with larger $N$.

An increase of the temperature produces a broadening of the PDF (although the tetrahedral shape is still preserved for small $T$ ). We present the same distributions for $T=10 \mathrm{MeV}$ in Fig. 7. The kinetic thermal energy is the responsible of making the average distances increase with temperature (in this case the average distance is computed as $1.03 \mathrm{fm}$ ), eventually preventing any kind of clustering among nucleons when the temperature dominates over the attractive $N N$ potential.

We remark that at temperatures of $T=120 \mathrm{MeV}$ we obtain no bound system for $N=4$ with the original Walecka potential $V_{A}$. The clustering of four nucleons (and the eventual formation of ${ }^{4} \mathrm{He}$ ) via strong $N N$ correlations requires a 

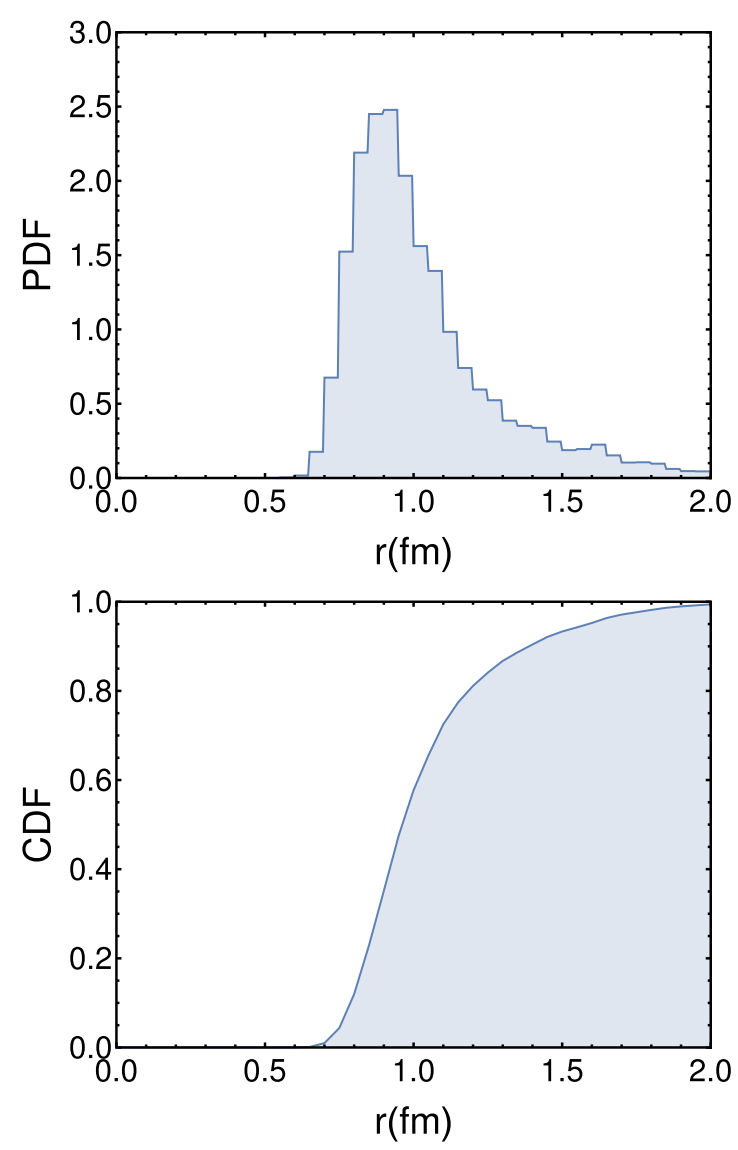

FIG. 7. Top panel: Histogram of the distance between nucleon pairs for $N=4$ simulation at $T=10 \mathrm{MeV}$. Bottom: Cumulative distribution function.

deeper potential for the freeze-out temperatures of baryon-rich HICs.

\section{2. $N=6:$ Octahedron}

The case with $N=6$ nucleons is still relatively simple to predict that the octahedron configuration will be the equilibrium shape. For $T=10^{-3} \mathrm{MeV}$ a fast equilibration is reached (see top panel of Fig. 8), sitting until $t=50 \mathrm{fm}$ in a metastable minimum of the potential (until the last particle is finally captured by the cluster). The final potential energy per nucleon is equal to $\langle V\rangle_{N}=-95.78 \mathrm{MeV}$, in agreement with Table II. A snapshot of the spatial configuration is presented in the lower panel of Fig. 8.

The distribution function of mutual distances is presented in the top panel of Fig. 9. It is possible to verify that the geometry is consistent with the expectations of an octahedron. This polyhedron has two different sets of relative distances, one at some distance $a$ and another at $\sqrt{2} a$ with relative strength 12 to 3 . This is precisely what we observe in the histogram, where the ratio between the area under the peaks is exactly 4 . This can alternatively be checked in the cumulative distribution function of the bottom panel of the same figure. The steps in this function are located at 0.848 and $1.199 \mathrm{fm}$, which correspond to the two distances between nucleons in the octahedron configuration. The minimum distance coin-
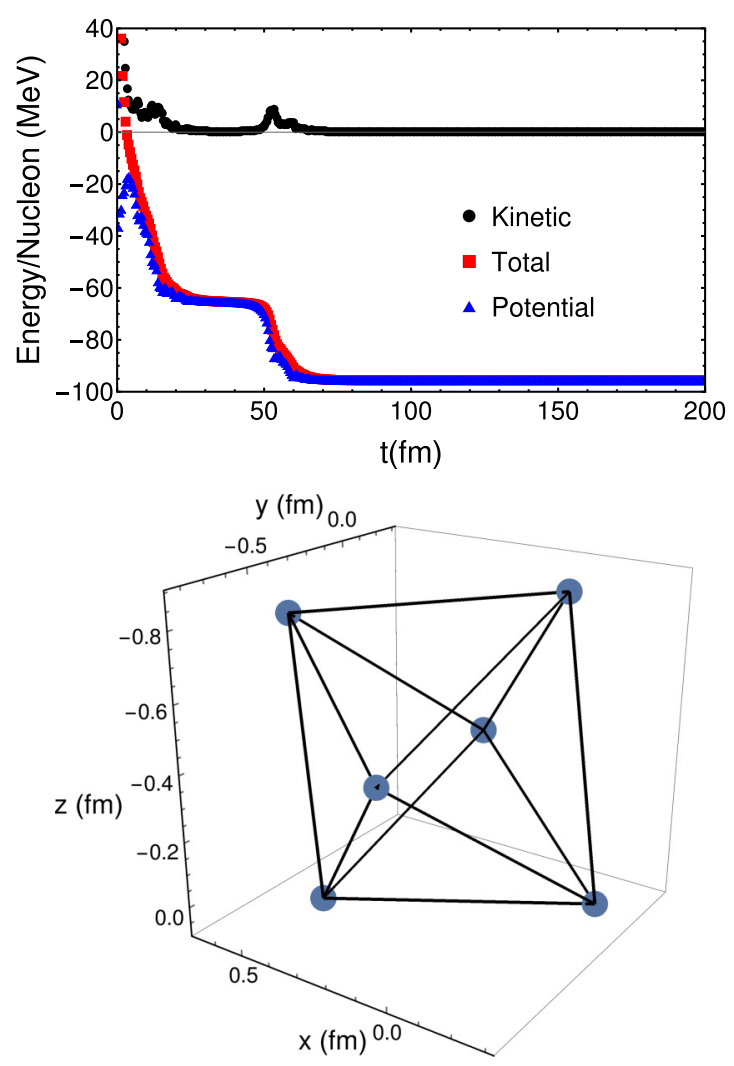

FIG. 8. Top: Kinetic (black), potential (blue), and total (red) energies per nucleon (in $\mathrm{MeV}$ ) for $N=6$ calculation vs time (fm) at $T=10^{-3} \mathrm{MeV}$. Bottom: Snapshot of the coordinate configuration at some time after equilibration.

cides with the expectations in Table II, and the second one is a factor $\sqrt{2}$ larger.

In Fig. 10 we can observe how already at $T=1 \mathrm{MeV}$ the two peaks are smeared out due to the thermal motion of the nucleons. Nevertheless, it is still possible to identify the octahedron configuration.

\section{3. $N=8$}

For $N=8$ we notice that the naive expectation of a cubic geometry was already ruled out in Sec. III B in favor of a square antiprism configuration. The later configuration has a lower potential energy for $N=8$ nucleons. The distribution of mutual distances is rather different from the cubic configuration case as seen in Table IV.

A calculation at finite temperature $T=1 \mathrm{MeV}$ seems to be roughly consistent with this expectation. The PDF shown in Fig. 11 is clearly inconsistent with a cubic configuration after comparing to the numbers in Table IV. To test the square antiprism configuration we run a calculation at $T=$ $10^{-3} \mathrm{MeV}$. The resulting PDF shown in Fig. 12 shows that this distribution is much richer and not consistent with this geometry. The potential energy per particle at $T=10^{-3} \mathrm{MeV}$ is $\langle V\rangle_{N}=-119.45 \mathrm{MeV}$, also not consistent with either cube of square antiprism (see Table II). We were not able to identify the precise geometrical shape (shown in the bottom panel of 

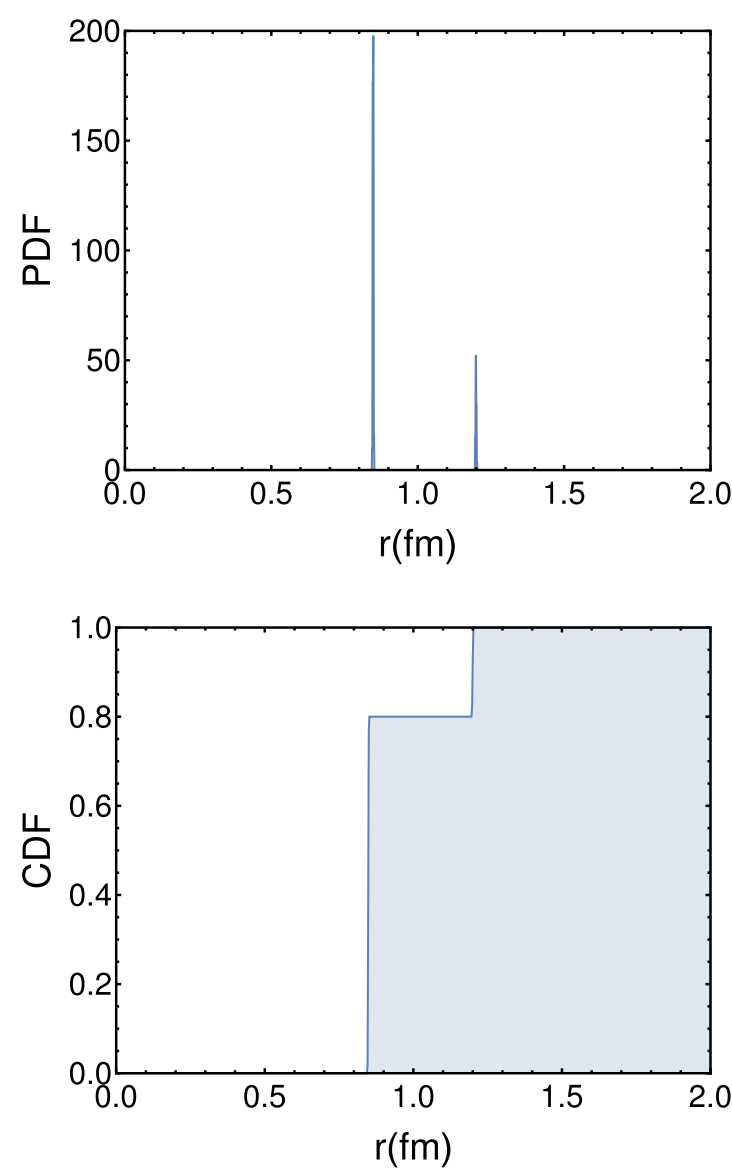

FIG. 9. Top: Histogram of the distance between nucleon pairs for $N=6$ simulation at $T=10^{-3} \mathrm{MeV}$. Bottom: Cumulative distribution function.

Fig. 12), but we have classified seven different distances with relative weights $2: 4: 1: 2: 1: 2: 2$.

\section{4. $N=12+1$ : Icosahedron +1}

We conclude the study of small clusters by considering $N=12+1$ nucleons at $T=10^{-3} \mathrm{MeV}$, where the expected configuration is an icosahedron plus one nucleon at the center. It is easy to see by naked eye that the geometrical configuration resembles this expectation. In the top panel of Fig. 13 we present a snapshot of the spatial configuration at some time after equilibration. In the middle panel we also present the distribution of (78) mutual distances. We observe four different sets of distances, and with a relative weight (see cumulative distribution function in the middle of the same figure) in excellent agreement with the expectations of Table IV. Finally, the minimum distance (position of the first peak of the distribution) is $0.782 \mathrm{fm}$, and the potential energy per nucleon obtained is $\langle V\rangle_{N}=-177.32 \mathrm{MeV}$, both in agreement with the values in Table II.

\section{Clustering at freeze-out temperatures}

In this section we describe simulations following the scheme presented in the previous section (MD + Langevin
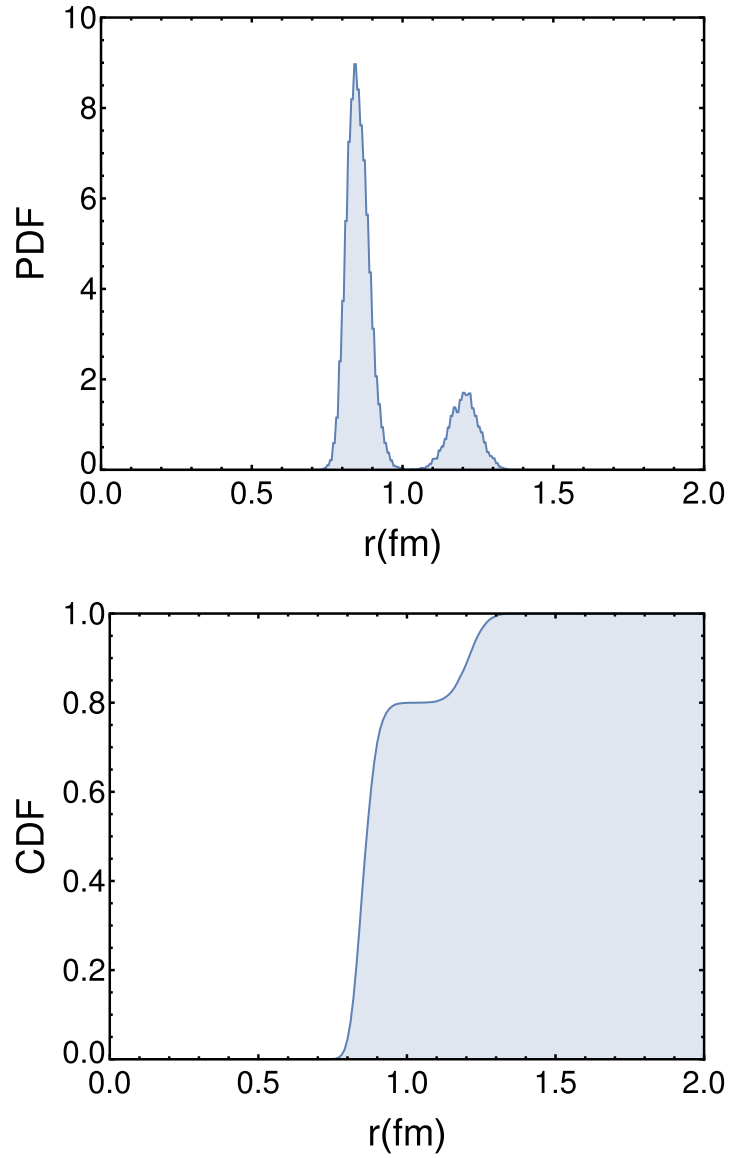

FIG. 10. Top: Histogram of the distance between nucleon pairs for $N=6$ simulation at $T=1 \mathrm{MeV}$. Bottom: Cumulative distribution function.

with modified Walecka potentials). The number of nucleons is larg, $N=128$, and the temperature is fixed at the typical freeze-out temperatures $T_{\text {kin }}=120 \mathrm{MeV}$ [21].

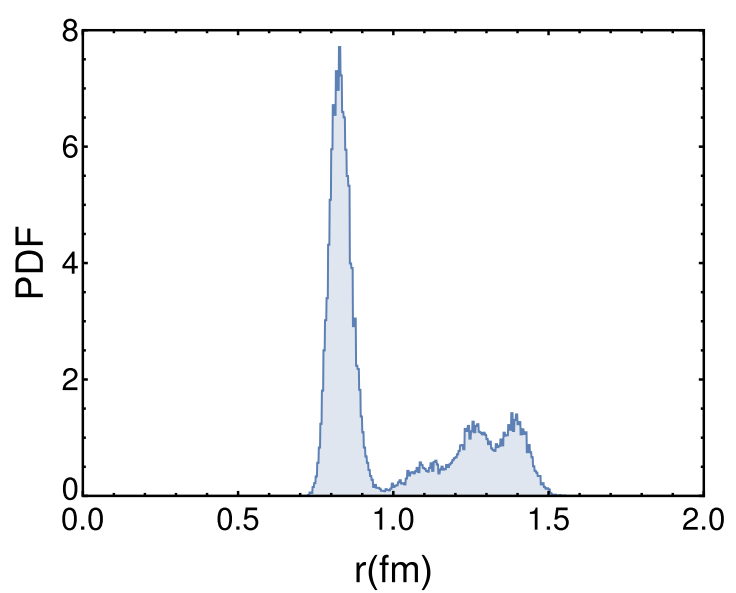

FIG. 11. Top panel: Histogram of the distance between nucleon pairs for $N=8$ simulation at $T=1 \mathrm{MeV}$. This configuration is apparently signaling a square antiprism shape, when comparing the distribution with the ideal distribution in Table IV. 

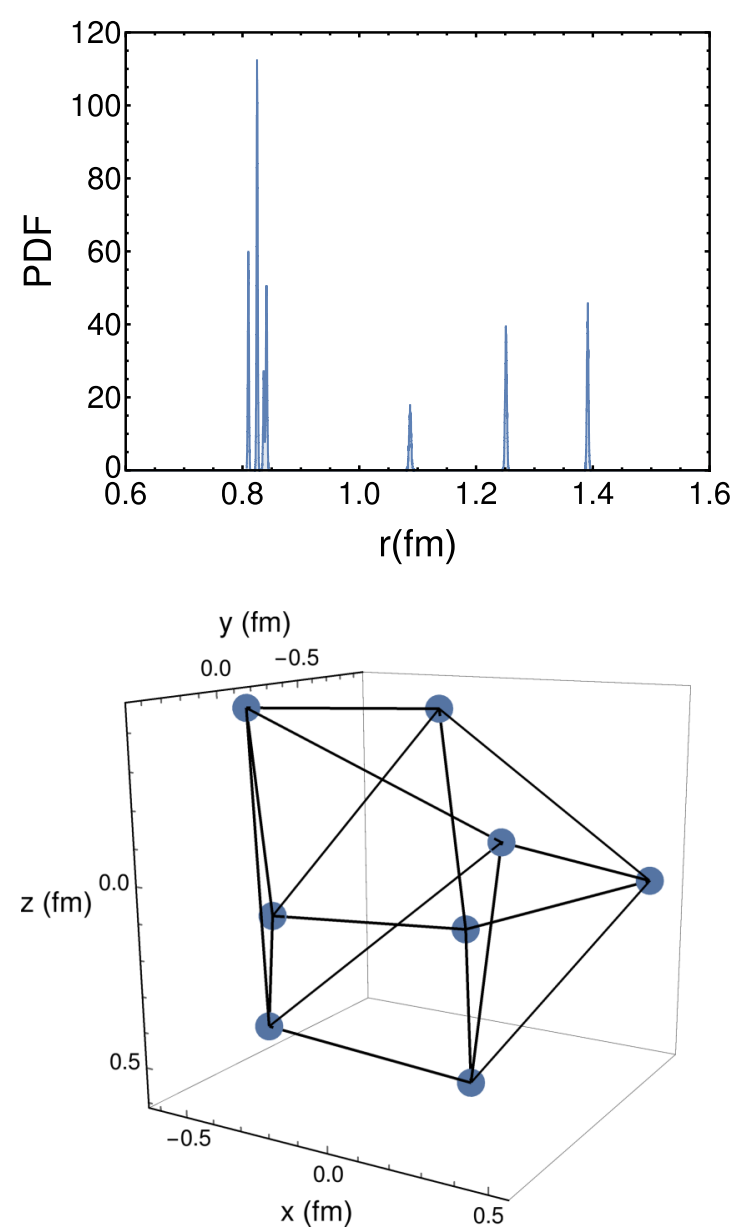

FIG. 12. Top: Histogram of the distances between nucleon pairs for $N=8$ simulation at $T=10^{-3} \mathrm{MeV}$. Bottom: Spatial configuration of the $N=8$ nucleons after equilibration.

In this section we use the potential $V_{A}$ to see how a deep potential can bind nucleons and eventually produce a large cluster. In this example we look for a clear example of such a cluster, and to apply various systematic procedures to analyze its internal structure. The initial state and a configuration after its equilibration are shown in Fig. 14. The initial geometry is spherical with a density of $n=0.16 \mathrm{fm}^{-3}$.

When equilibrium is reached we obtain a big cluster which includes all $N=128$ particles. In Fig. 15 we show the time evolution of the kinetic, potential, and total energies (top) as well as the temperature evolution (bottom). We observe that the total energy of the system is dominated by large negative potential energy, so to see one cluster structure is not surprising. The temperature at equilibrium (plateau formed after $t \sim 50 \mathrm{fm}$ in the bottom panel) fluctuates around the value $T_{\text {kin }}=120 \mathrm{MeV}$. The sudden kicks in temperature and the steps in energies occur when one more particle is captured by the cluster, and falls to the deep potential well.

Following the mean-field approach and the King's solution a decreasing distribution of particles is expected as a function of the radial distance. We want to analyze the internal arrangement of nucleons in this cluster by finding the radial distribution of nucleons starting at $r=0$ (defined as the
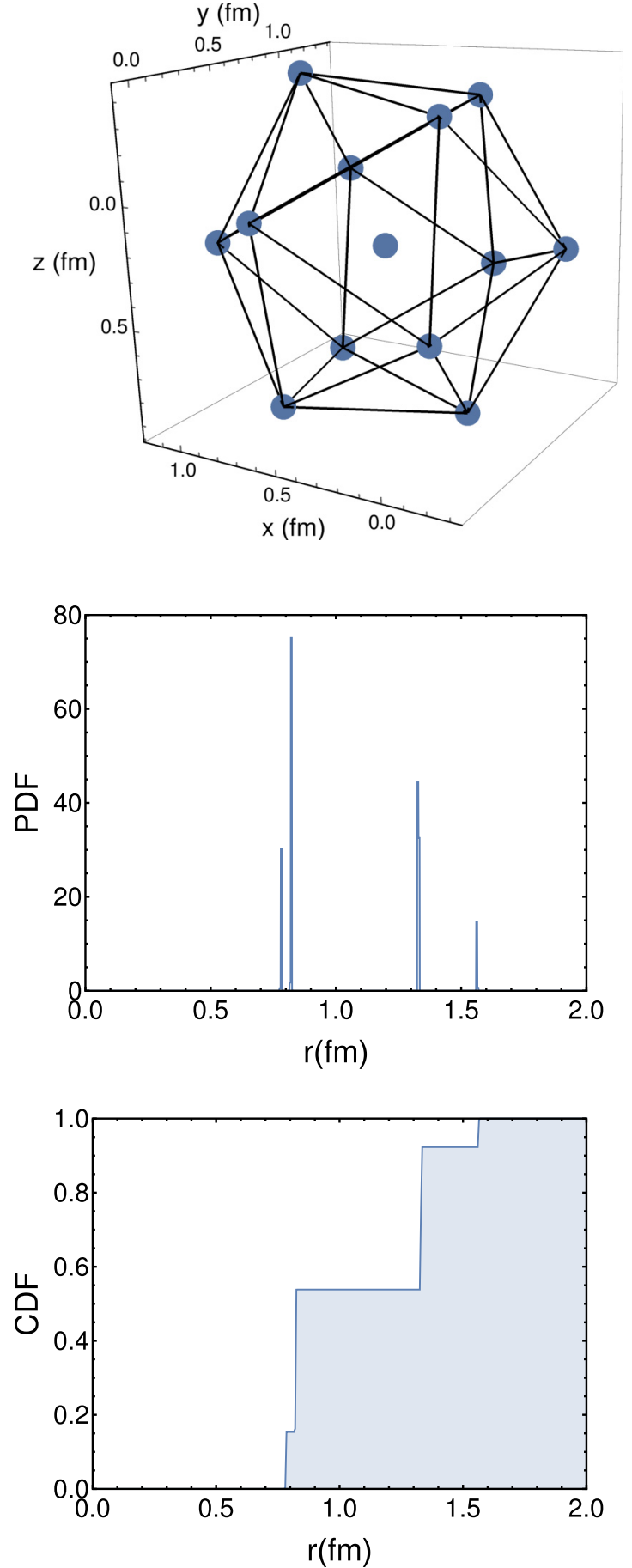

FIG. 13. Top: Spatial configuration of $N=13$ nucleons at $T=$ $10^{-3} \mathrm{MeV}$ at some arbitrary time after equilibration. Middle: Histogram of the distances between nucleon pairs for the same simulation. Bottom: Cumulative distribution function.

centroid of the cluster). As the centroid evolves in time, we monitor its position at each time step, and perform the radial distribution of the nucleons. To have independent events in the distribution, and to avoid spurious correlations we choose time steps well separated to perform the average. We measure the number of nucleons per unit volume/radial distance, and plot it versus the distance from the centroid. 

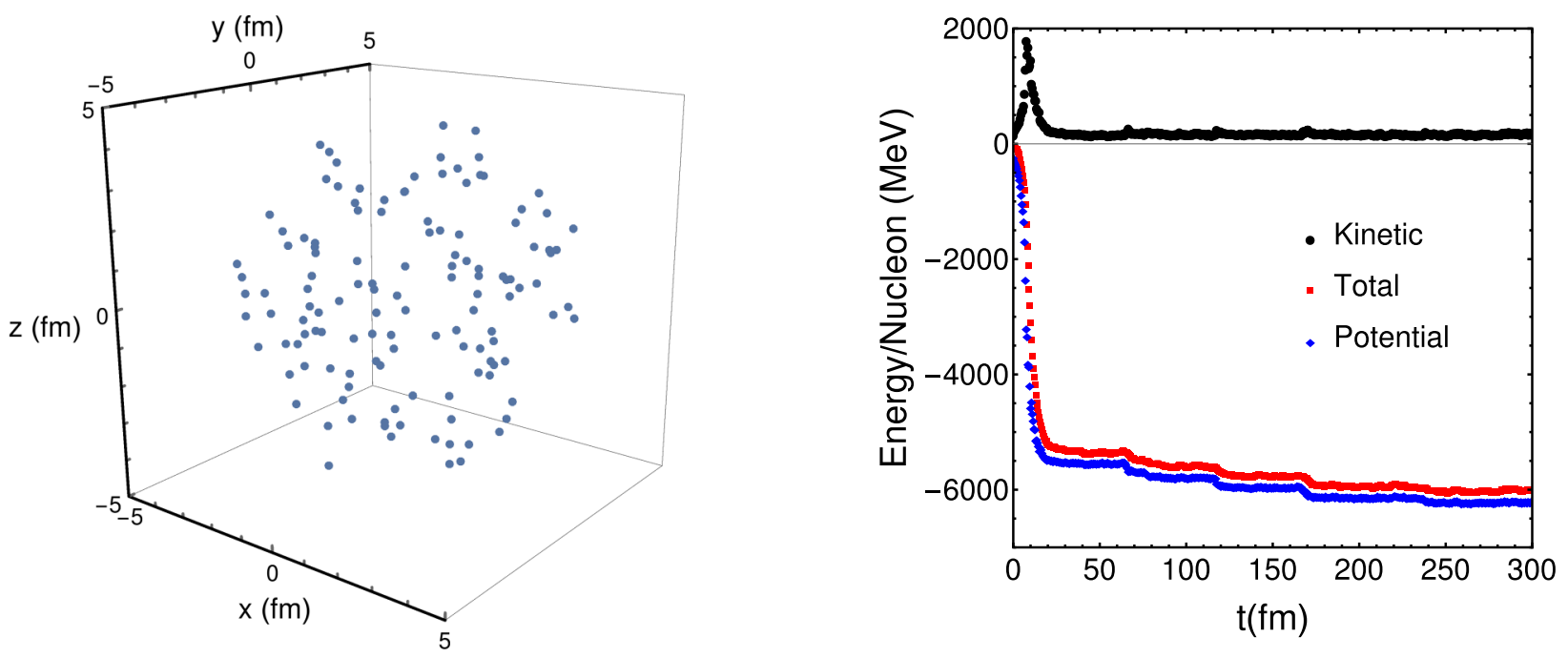

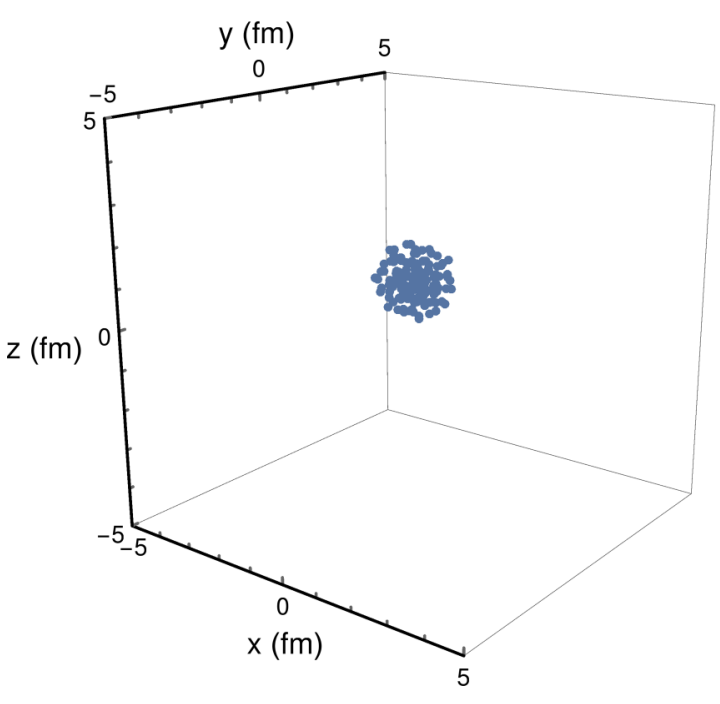

FIG. 14. Configuration of $N=128$ nucleons in coordinate space at initial time $t=0 \mathrm{fm} / c$ (top) and at some arbitrary time after full equilibration $t>250 \mathrm{fm} / c$ (bottom).

The distributions $d N / d V$ and $d N / d r$ are represented in Fig. 16 showing a nonmonotonous structure suggesting a shell-like organization with accumulations of nucleons every $0.3 \mathrm{fm}$ in the radial direction.

For clarity, let us note that this study is only done for investigative purposes. The time scales considered in the plot above, up to $t \sim 300 \mathrm{fm} / c$, are much longer than those available in heavy-ion collisions, $t \sim 10 \mathrm{fm} / c$. Furthermore, this analysis was done in static, rather than exploding, heat bath. So, by no means do we suggest that such heavy clusters are actually produced in experiment. At best we hope to find evidence of the very beginning of the clustering process.

\section{BARYONIC CLUSTERS NEAR THE CHIRAL TRANSITION}

In this study we continue the study of big cluster formation and their time scales as the clustering process becomes more and more important. This will happen when the original

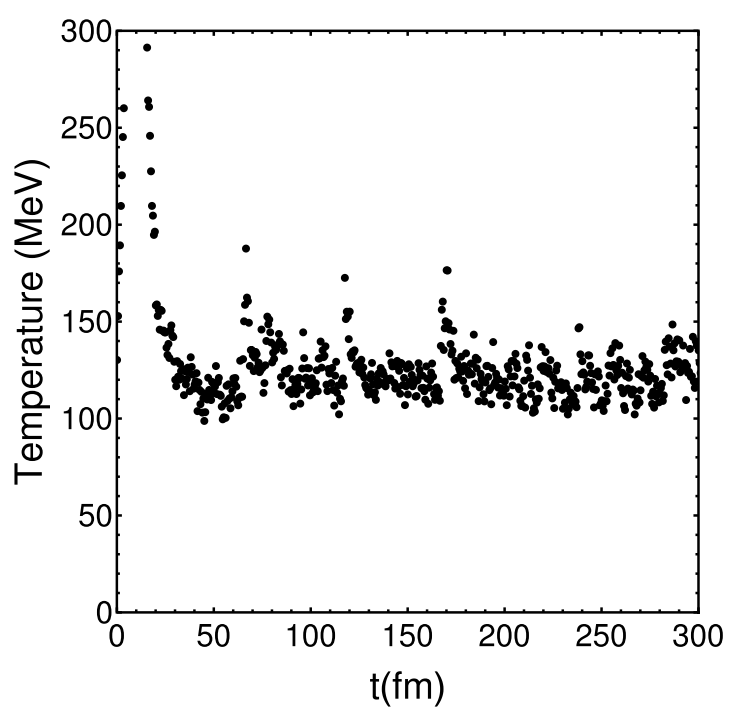

FIG. 15. Top: Kinetic (black dots), potential (blue diamonds), and total energies (red squares) as a function of time for a configuration of $N=128$ nucleons using the Walecka potential $V_{A}$. Bottom: Temperature as a function of time for the same simulation.

parameters of the Walecka potential are modified as a consequence of the changes in the properties of the $\sigma$ mode.

We will compare the potentials $V_{B 1}, V_{B 2}, V_{C}$, each one thought to be acting closer and closer to the chiral transition.

\section{A. Formation of clusters}

All simulations begin with randomly placed nucleons. Naturally the cluster formation starts with small clusters, which then assemble into larger and larger ones. We decided to follow the process by defining variables in which one can separate clustered and nonclustered baryons in the most direct way, and then histogram the distributions at different times.

We performed a number of such studies, demonstrating here one example for a four-particle variable. The variable $S$ (from sum) is defined as the normalized sum of all mutual 

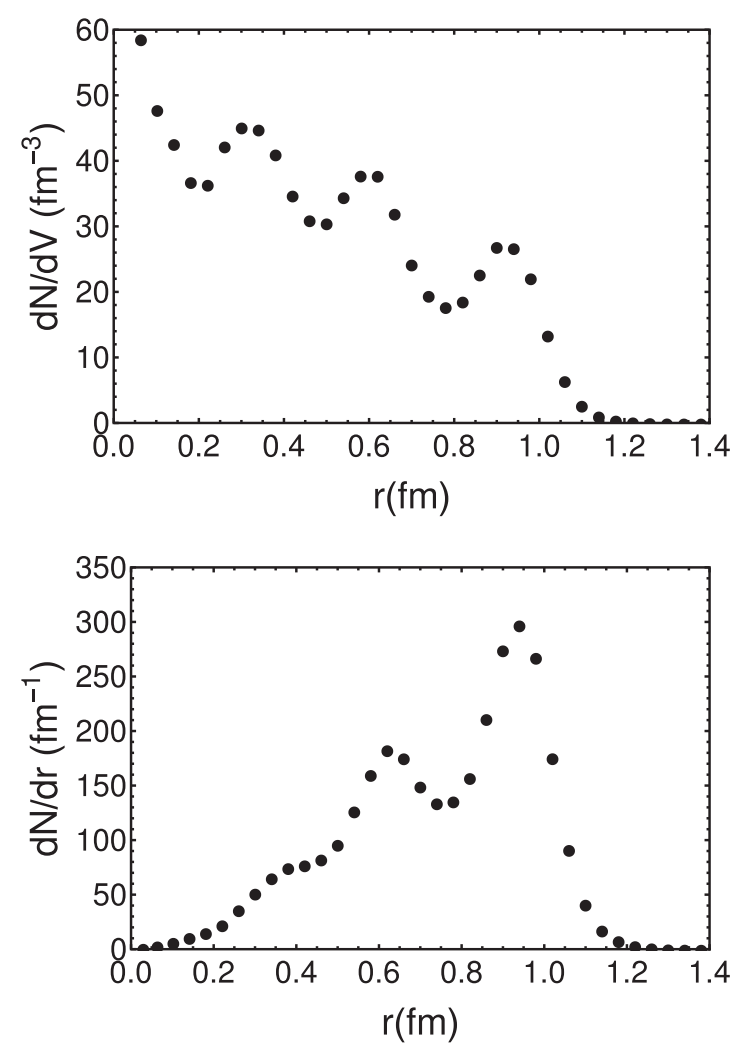

FIG. 16. Density of nucleons per unit volume (top) and per unit distance (bottom) inside a cluster of $N=128$ nucleons after equilibration.

distances between particles in the system,

$$
S=\frac{1}{N_{d}} \sum_{\substack{i=1 \\ j>i}}^{N_{d}}\left|\vec{x}_{i j}\right|,
$$

where $i, j=1, \ldots, N$ run over all nucleons, $\vec{x}_{i j}$ is the vector joining pairs, and $N_{d}=N(N-1) / 2$ is the number of mutual distances between different nucleons.

As one can see from an example shown in Fig. 17 (note the scales), for potentials $A, B 1, B 2$ we observe that the entropy wins over the energy. With time the distribution slowly becomes wider due to the diffusion of baryons.

In contrast to that, the potential $C$ with longer-range attraction shows the opposite trend, the potential wins over the entropy, leading to a rather robust clustering. An example of the time evolution for the $C(x=1)$ potential is shown in Fig. 18. The clear separation of the distribution into two peak structure, in this one particular event, corresponds to a formation of two clusters (in this event, those have sizes of 9 and 22, with only one particle evaporating out). The first peak corresponds to intracluster distances in both clusters, whereas the second peaks reflect intercluster distances.

\section{B. Time scales}

We consider a system of $N=128$ nucleons at temperature $T=80 \mathrm{MeV}$, with an initial nuclear density $n=0.13 \mathrm{fm}^{-3}$
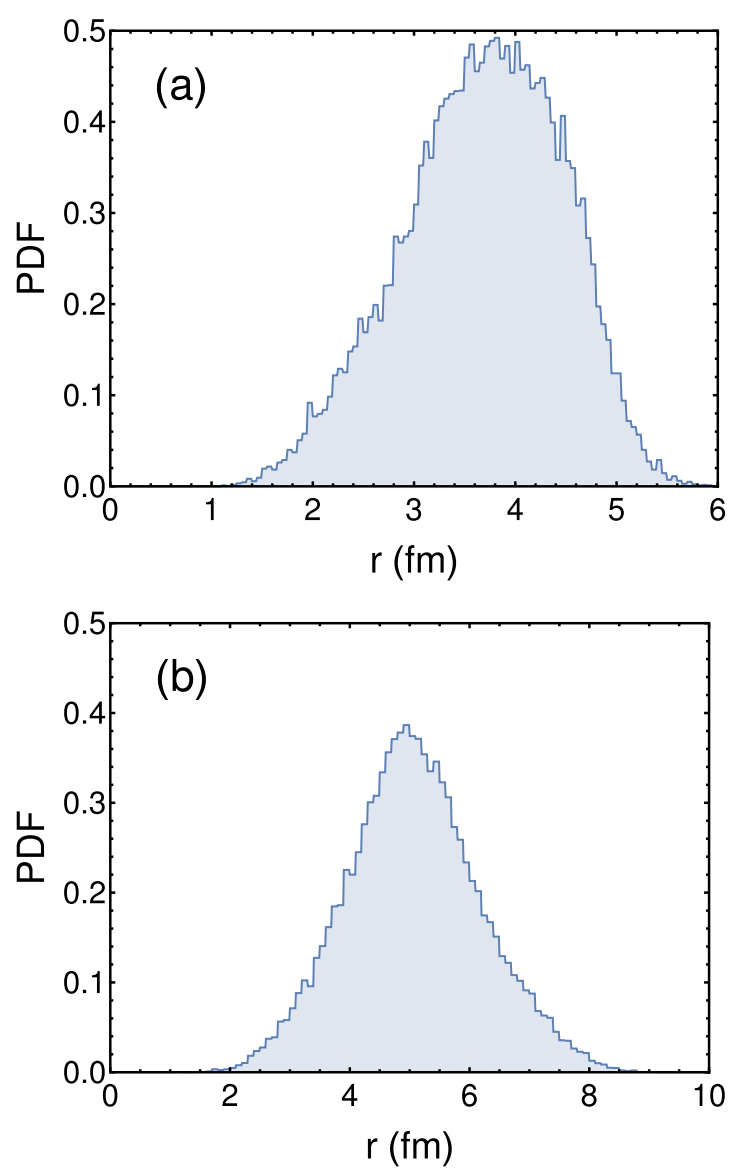

FIG. 17. Distribution over variable $S$ Eq. (28), for time equal $t=0$ (a) and $4 \mathrm{fm} / c$ (b). The calculation is done for $N=32$ particles and the $V_{A^{\prime}}(r)$ potential.

and finite size. In Fig. 19 we show the time dependence of the energies per particle (top) and the temperature (bottom). After a fast thermal equilibration the temperature is approximately constant, while the total energy is not conserved in the evolution as dissipation occurs due to the drag force.

The potential $V_{B 1}$ is able to produce full clustering after long times. From the example in Fig. 19, the full equilibration time is of the order of $\sim 800 \mathrm{fm} / c$, and clustering has taken place. Individual particles can escape the cluster thanks to their kinetic energy, however, we avoid the loss of particles with the external trapped potential in Eq. (22).

We can define an equilibration time by noticing that the total energy has an approximate exponential decay exp $\left(-\frac{t}{t_{\text {eq }}}\right)$. We obtain $t_{\text {eq }}=187 \mathrm{fm}$ in this particular example.

Although the $V_{B 1}$ is enough to form a big cluster in several hundreds $\mathrm{fm} / c$, these scales are totally irrelevant for HICs. A slightly critical potential $V_{B 2}$ produces the clustering in a much faster way, $\sim 40 \mathrm{fm} / c$. We present the time dependence of the energies and temperature in Fig. 20.

In this case, the exponential decay is much less evident. We find an initial regime of $\sim 10 \mathrm{fm} / c$ where the energy is approximately constant. Between 10 and $17 \mathrm{fm}$ we find a good exponential decay with an equilibration time of $t_{\mathrm{eq}}=3 \mathrm{fm} / c$. After this transient exponential decay the relaxation is much softer, reaching equilibration in around $40 \mathrm{fm} / c$. The time 

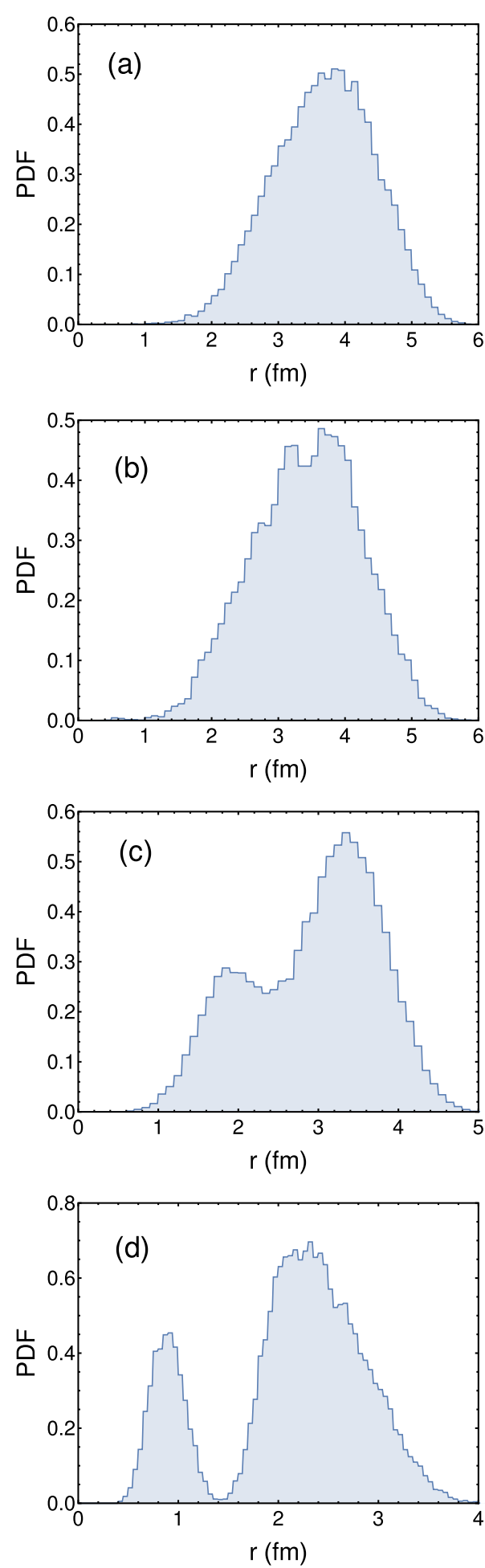

FIG. 18. Distribution over variable $S$ Eq. (28), for time equal $t=0,1,2,4 \mathrm{fm} / c$, in panels (a)-(d), respectively. The calculation is done for $N=32$ particles and the $V_{C}(r ; x)$ potential with $x=1$.

scales for the clustering with this potential are much closer to those in heavy-ion collision, so it seems reasonable to consider this mechanism as potentially important close to the critical point (where the equilibration time is even reduced using a deeper potential like $V_{C}$ ).
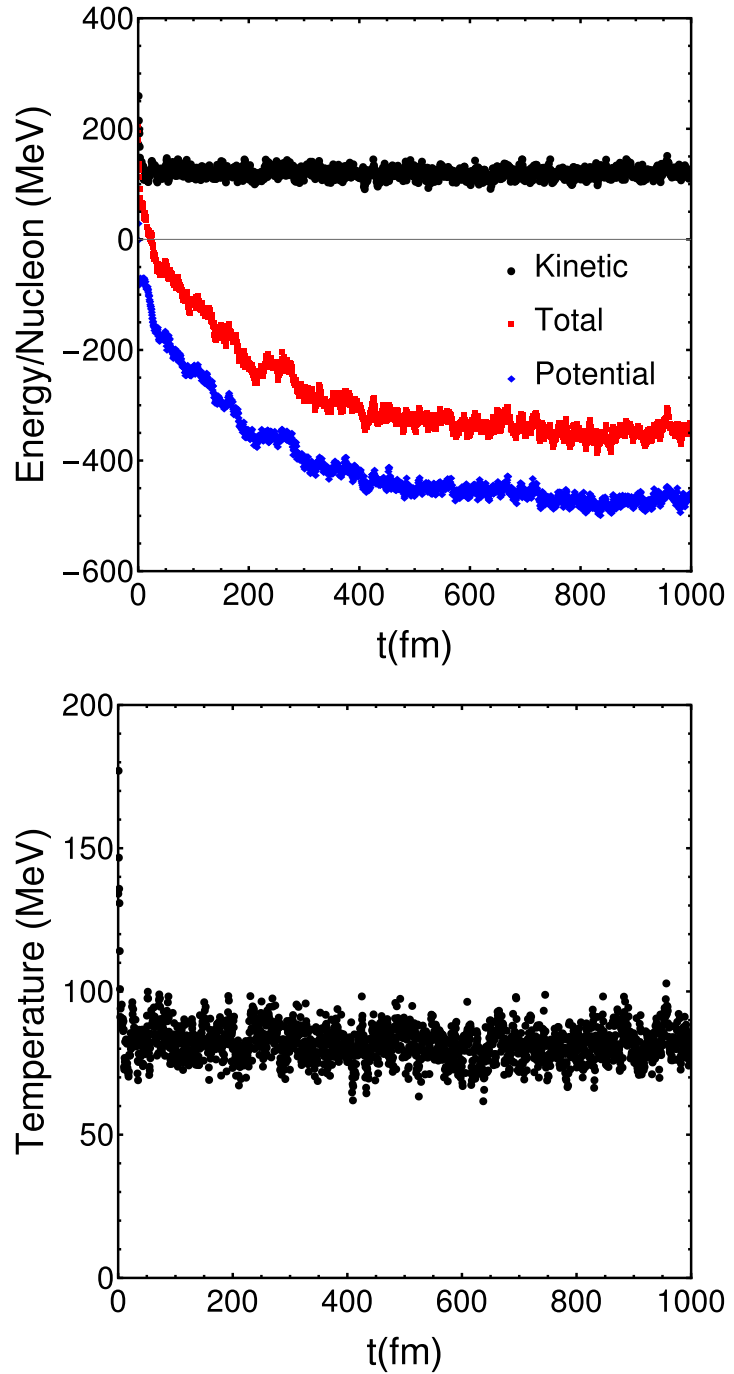

FIG. 19. Top: Kinetic (black dots), potential (blue diamonds), and total energies (red squares) as a function of time for a configuration of $N=128$ nucleons using the potential $V_{B 1}$. Bottom: Temperature as a function of time for the same simulation.

Nevertheless, it seems clear that the time for full clustering is still large to take place completely in heavy-ion collisions. We only hope to have a potential effect close to the critical point where the signatures of initial clustering might certainly occur (perhaps clusters of few nucleons as ${ }^{4} \mathrm{He}$ ). Starting from a system away from the critical point, we will calibrate our model with noncritical potential $V_{A}$ to experimental data at energies where Poissonian fluctuations are observed. Then, we will modify our potential to increase criticality and compute observables like higher-order moments of the (net-)proton distribution.

\section{RESULTS: CORRELATION AND CLUSTERING AT BES CONDITIONS}

In this section we apply our model to heavy-ion collisions at the BES conditions. Due to the practical limitation of our model, we will not perform a full comparison to experimental 

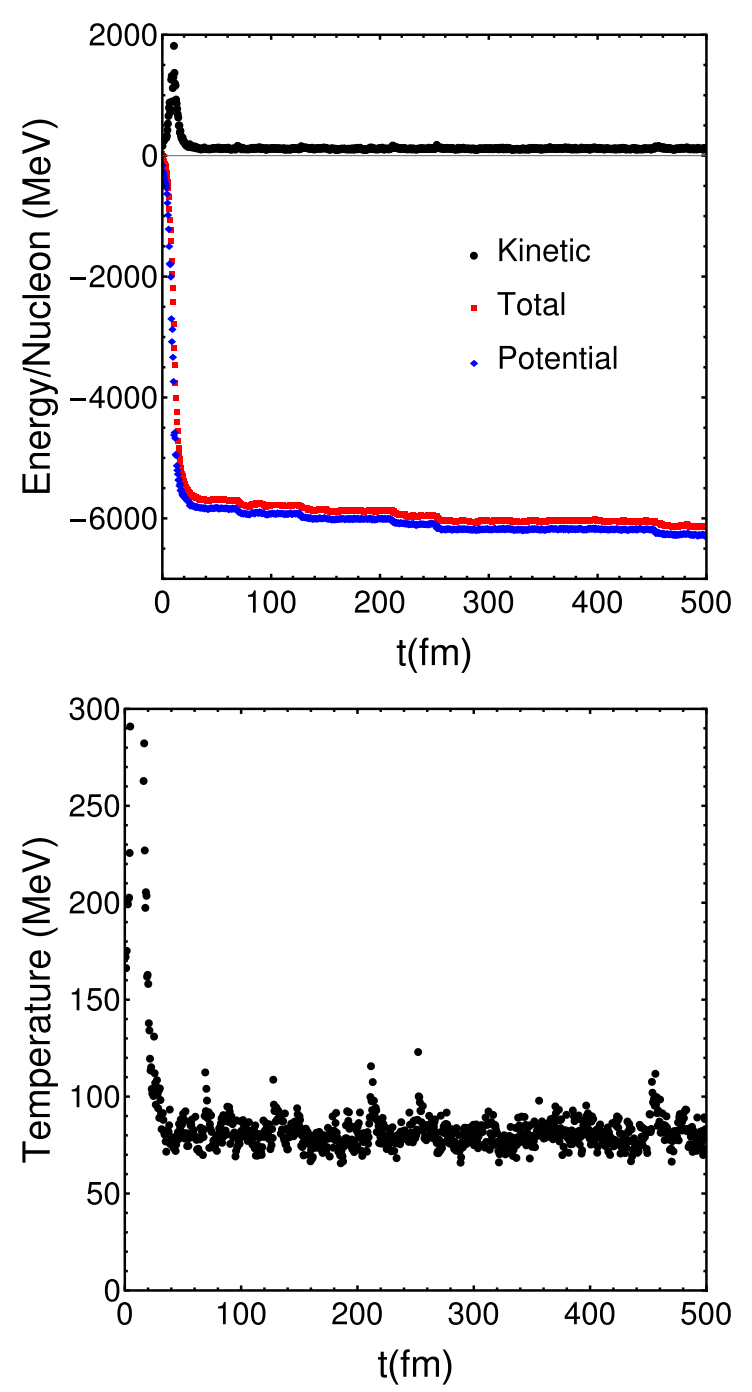

FIG. 20. Top: Kinetic (black dots), potential (blue diamonds), and total energies (red squares) as a function of time for a configuration of $N=128$ nucleons using the potential $V_{B 1}$. Bottom: Temperature as a function of time for the same simulation.

data. Rather than providing a complete quantitative answerfor what one would need a more sophisticated dynamical code-we content ourselves to show that the obtained effect (using the closest conditions to experiment we are able to implement) is consistent with what is observed in the laboratory.

At beam collision energies above $\sqrt{s_{N N}}=19.6 \mathrm{GeV}$, STAR data have shown a very approximate Skellam distribution for net protons [5,22], consistent with thermal equilibrium fluctuations. Therefore, we will choose the energy $\sqrt{s_{N N}}=19.6 \mathrm{GeV}$ as our baseline, where thermal effects dominate over the interaction potential.

For collisions at this energy (and lower) we can safely neglect antiprotons. In Table $\mathrm{V}$ we show the ratio of protons/antiprotons in the kinematic cut $|y|<0.5,0.4<p_{\perp}<$ $0.8 \mathrm{GeV} / c$ for the most central collisions at the different energies considered in this work. Data are adapted from Ref. [22].
TABLE V. Proton-to-antiproton ratio for $|y|<0.5$ and $0.4<$ $p_{\perp}<0.8 \mathrm{GeV} / c$ at centrality bin $0-5 \%$ for collision energies $\sqrt{s_{N N}} \leqslant 19.6 \mathrm{GeV}$. Adapted from Ref. [22].

\begin{tabular}{lccc}
\hline \hline$\sqrt{s_{N N}}(\mathrm{GeV})$ & 7.7 & 11.5 & 19.6 \\
\hline Proton/antiproton & $114.4 \pm 0.6$ & $30.64 \pm 0.07$ & $9.89 \pm 0.01$ \\
\hline \hline
\end{tabular}

In our own simulations, Poisson statistics is achieved when measuring the distribution of protons in a subvolume or the order of few percent of the initial volume. This is consistent with the fact that the experimental net-proton distribution in the mentioned $p_{T}$ cut is $5 \%$ of the total net-proton multiplicity [23]. In that case the protons follows very closely the Poisson expectations.

Coming back to our code, our first task is to achieve a similar particle distribution in such a noncritical scenario, where Poissonian fluctuations dominate. We identify the potential $V_{A^{\prime}}$, i.e., the Walecka potential with additional repulsion (and no critical enhancement), with the scenario at $\sqrt{s_{N N}}=$ $19.6 \mathrm{GeV}$.

The parameters are chosen as follows. The kinetic freezeout temperature for these collision energies is roughly $T_{\text {kin }}=$ $120 \mathrm{MeV}$. Our code will simulate a few Fermi/c between hadronization and freeze-out, so we have set a constant temperature to $T=150 \mathrm{MeV}$. We have observed that the calculation is not very sensitive to a change of temperature around this value.

The baryon density at freeze-out is close to $n_{\text {kin }} \sim$ $0.12 \mathrm{fm}^{-3}$, but at earlier stages it can take a few times this value [24]. We will use a value of $n=0.3 \mathrm{fm}^{-3}$. We run our $\mathrm{MD}+\mathrm{L}$ code for a time of $\Delta t=5 \mathrm{fm} / c$, corresponding to an approximate time between hadronization and kinetic freezeout. While this time could be safely extended, perhaps up to a factor 2, we prefer to be conservative not to overestimate the effect of clustering (as seen in previous sections).

One does not know how many protons (on average) the physical system contains in the full volume. What one does know is the average number of protons in a certain kinematic cut. Therefore, one should fix the total number of protons $N$ in the simulation in such a way that this average is achieved. For numerical convenience we simply use a reduced number of protons $N=32$ and then scale up the different cumulants as suggested in Ref. [25] to be able to compare with the experimental one. It is important to note that the scaled skewness $S \sigma$ and kurtosis $\kappa \sigma^{2}$ do not depend on the volume, so we can directly compute them without the need of any multiplicative factor. However, to be able to compare the cumulants themselves it is mandatory to select the appropriate number of protons in the system.

The number of events for each energy (or $N N$ potential) is $N_{\mathrm{ev}}=10^{5}$. This number is of the same order of the experimental number of events for the largest centrality bin. While one is not required to implement the same number of events as in experiment (as long as it is large enough to provide accurate results), we opt to do this to perform an additional check when comparing the magnitude of the statistical uncertainties with the experimental ones. 
TABLE VI. Parameters used in the simulations of protons for kinetic freeze-out conditions of STAR collisions at $\sqrt{s_{N N}} \leqslant 19.6 \mathrm{GeV}$. Respectively: temperature, nucleon density, number of nucleons, number of events, drag coefficient, and time duration.

\begin{tabular}{lccccc}
\hline \hline$T$ & $n$ & $N$ & $N_{\mathrm{ev}}$ & $\lambda$ & $\Delta t$ \\
\hline $150 \mathrm{MeV}$ & $0.3 \mathrm{fm}^{-3}$ & 32 & $10^{5}$ & $0.256 \mathrm{fm}^{-1}$ & $5 \mathrm{fm} / c$ \\
\hline \hline
\end{tabular}

As explained in Sec. $\mathrm{V}$ the value of the dissipative force $\lambda$ is taken from the results of Ref. [19].

We summarize the parameters used in this section in our $\mathrm{MD}+\mathrm{L}$ simulations in Table VI. For the aim of isolating the effect of the $N N$ potential, these parameters will be kept fixed for all potentials (all collision energies). We checked that from the thermal fits of the different energies, these parameters do not vary too much (the most sensible parameter is the baryochemical potential).

The calculation is performed in a nonexpanding medium, i.e., without radial flow implemented. This is convenient for the use of nonrelativistic dynamics at all times. A final boost in rapidity and transverse momentum will take care of the mapping of the particles into the appropriate kinematic domain, consistent with experiment. We will address this step later.

The initial conditions of the simulations are in order. Due to the longitudinal expansion of the fireball, the geometry is approximately cylindrical. We initialize a volume with a height of $h=8 \mathrm{fm}$ and a radius of $R=\sqrt{\frac{N}{n h \pi}}$ [24]. The nucleons are initialized randomly in coordinate space. From the statistical thermal model we know that a thermal distribution in equilibrium is a good approximation, at least for the noncritical regime. Therefore we will initialize the momenta according to a Boltzmann distribution at a temperature $T$.

We now describe the outcome of this simulation with $V_{A^{\prime}}$ and how we implement the effects of the expansion to match the measured distributions. For the noncritical potential, collisions with light particles (through the stochastic forces) are dominant, and the system maintains equilibrium. After the simulations we obtain Gaussian distributions for rapidity and transverse momentum, expected for a nonrelativistic gas in equilibrium. Moreover, the width of the Gaussian are perfectly consistent with the temperature used.

Because of this the rapidity distribution,

$$
\frac{d N}{d y} \propto e^{-y^{2} m_{N} / 2 T},
$$

is relatively narrow around $y=0$, and it provides a large fraction of protons at midrapidity. This is not consistent with experiment, where one expects $\sim 10 \%$ of particles at midrapidity $|y|<0.1$, and the distribution should be almost uniform between the limiting rapidities. As already stated, the reason for this discrepancy is that our simulation does not account for the longitudinal expansion of the system. To account for the fireball expansion, we perform a final mapping of the $\left(y, p_{\perp}\right)$ variables to mimic the experimental distributions.

We transform the Gaussian $y$ distribution into a uniform distribution, which should be much closer to reality. The

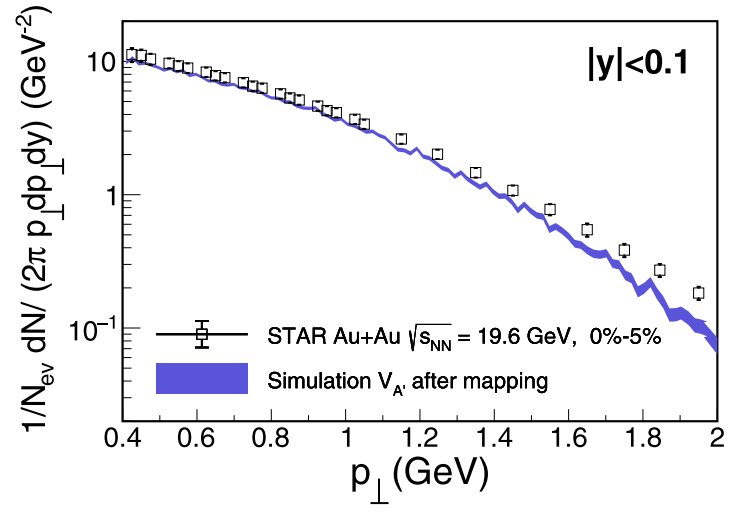

FIG. 21. Transverse momentum distribution for protons in a lowenergy heavy-ion collision. The data correspond to the STAR results at $\sqrt{s_{N N}}=19.6 \mathrm{GeV}$ for collisions in the $0-5 \%$ centrality class at midrapidity $|y|<0.1$. The band is our result from $10^{5}$ simulations using the noncritical $V_{A^{\prime}}$ potential after the mapping of the kinematic variables $y, p_{\perp}$ to account for the fireball expansion.

transformation is given by

$$
y \rightarrow y^{\prime}=y_{\min }+\frac{y_{\max }-y_{\min }}{2} \operatorname{Erfc}\left(-y \sqrt{\frac{m_{N}}{2 T}}\right),
$$

with the limiting beam rapidities $y_{\min }$ and $y_{\max }$. The distribution of $y^{\prime}$ is $U\left(y_{\min }, y_{\max }\right)$.

The experimental $p_{\perp}$ distribution of net protons is well fitted to a double exponential [21]. We have found that for the considered kinematic cut a Gaussian form is already very accurate to describe the data, but with a broader width than the one we obtain. Therefore, by simply rescaling the transverse momenta of our particles by a factor of 1.7 we can match the experimental distribution with reasonable accuracy.

To compare the final distribution in $p_{\perp}$ with the experimental one, one needs to find the overall normalization of the distribution (due to our use of $N=32$ ). In this respect we multiply our $p_{\perp}$ distribution by a factor 6.6 , which will be justified below.

The resulting distribution is shown in Fig. 21 compared to the proton distribution measured experimentally. The reasonable agreement allows us to move on to more local observables.

We proceed to analyze the proton distributions by performing the two kinematical cuts considered in the literature. In what follows we will denote cut 1 as the one with rapidity $|y|<0.5$ and transverse momentum $0.5 \mathrm{GeV} / c<p_{\perp}<$ $0.8 \mathrm{GeV} / c$, whereas cut 2 extends the $p_{\perp}$ coverage up to $2 \mathrm{GeV} / c$, thanks to the time of flight detector for the particle identification [5].

We summarize our results for the proton cumulants in Fig. 22 compared to experimental data for the two cuts. In our simulation we perform the analyses with $N_{\mathrm{ev}}=10^{5}$ events. The statistical uncertainty comes from the Delta theorem, as explained in Ref. [26]. As mentioned before, we need to fix the overall normalization from our $N=32$ to match the absolute number of protons observed in experiment. To do this we choose the average number of protons $C_{1}$ in cut 1 and scale up our value of $C_{1}$ to match the experimental result. The factor is 

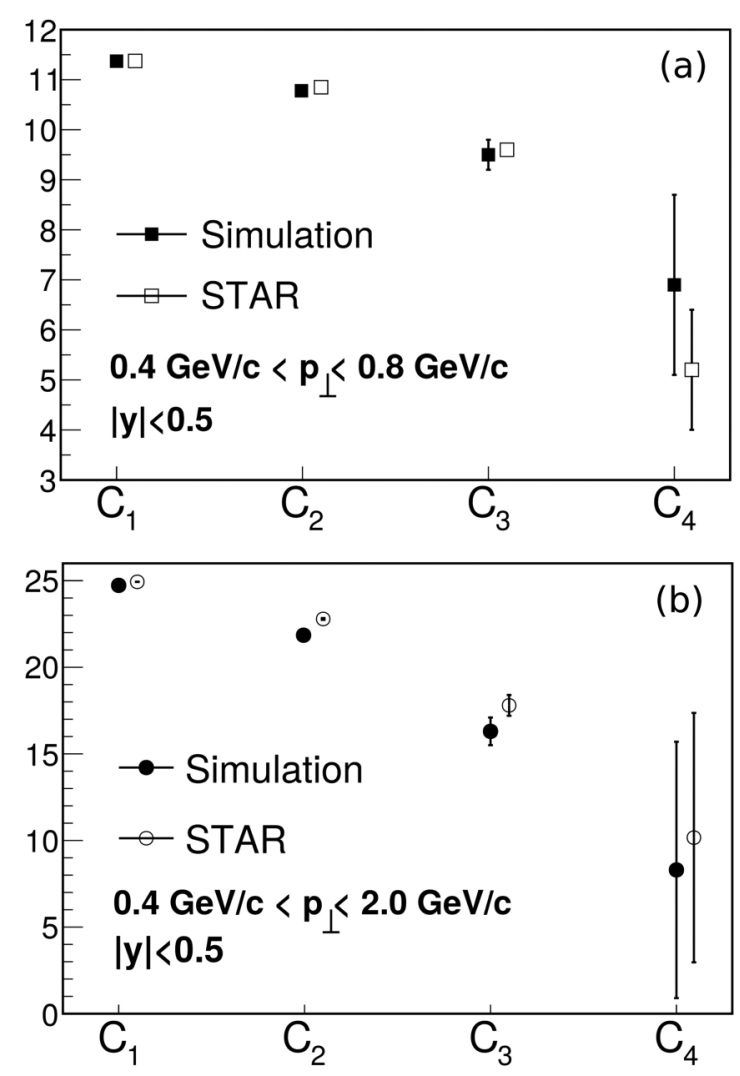

FIG. 22. Mean and central moments for proton distribution $\left(C_{2}\right.$ is the variance) for two different kinematic cuts at kinetic freeze-out conditions of STAR collisions at $\sqrt{s}_{N N} \leqslant 19.6 \mathrm{GeV}$. Our simulation results have been scaled by a constant factor 6.6, which is fixed by matching the experimental value of $C_{1}$ in cut 1 [upper left point in (a)]. Experimental data for cut 1 (a) is taken from [22] and for cut 2 (b) from [5].

found to be 6.6. Then, all the other cumulants (up to fourth order) are scaled up by the same amount [27]. This is the factor that we used to compare our $p_{\perp}$ distribution with the experimental one in Fig. 21.

We observe that despite our simple model, we can match in a good degree of accuracy the cumulants for proton number in both kinematical cuts for $\sqrt{s_{N N}} \leqslant 19.6 \mathrm{GeV}$. We can say that the noncritical scenario is reasonably under control.

\section{A. Proton skewness and kurtosis at freeze-out}

After fixing the parameters, the overall multiplicity normalization, and the $\left(y, p_{\perp}\right)$ mapping to account for the fireball expansion, we are ready to present our results for the higherorder moments of the proton distribution. We will show our results as functions of the $N N$ potential. No other simulation parameter is changed in what follows.

By computing ratios of the obtained cumulants, one can access the scaled skewness $S \sigma=C_{3} / C 2$ and kurtosis $\kappa \sigma^{2}=$ $C_{4} / C_{2}$ for protons. We will compare our outcome with the experimental net-proton skewness and kurtosis. As indicated before, cf. Table V, one should bear in mind a systematic uncertainty due to the absence of antiparticles in our model, representing roughly a $10 \%$ of systematic error at $\sqrt{s_{N N}}=$ $19.6 \mathrm{GeV}$ (becoming much smaller for lower energies).

We perform a total of $N_{\mathrm{ev}}=10^{5}$ events for each of the nuclear potentials at our disposal. Each of them encodes the modification of the $N N$ interaction due to the closer presence of the QCD critical point. Starting from our baseline at $\sqrt{s_{N N}}=19.6 \mathrm{GeV}$ with the noncritical potential $V_{A^{\prime}}$, a decrease of the collision energy is associated to an increase of the internucleon attraction. We will subsequently apply the potentials $V_{A^{\prime}}, V_{B 1}, V_{B 2}$, and $V_{C}(x)$ with $x=0.1,0.5,1$. Notice that a precise matching between the experimental collision energy and the particular $N N$ potential is not possible without extra modeling. Therefore we cannot directly compare our results with real data, but nevertheless one can observe the qualitative effect on the skewness and kurtosis after increasing the criticality in our model.

In Fig. 23 we our present our results for $S \sigma$ and $\kappa \sigma^{2}$. From top to bottom we show the theoretical skewness as a function of the $N N$ potential, the experimental skewness as a function of the collision energy, and the same dependencies for the kurtosis, respectively. In all cases we consider both cut 1 and cut 2. As mentioned, a direct comparison is not possible due to the difficulty of matching a given potential to a precise collision energy. However, we base our study on the idea that lowering the collision energy from high energies should necessarily approach the expanding system to the critical region, until some particular value of $\sqrt{s_{N N}}$. In our setup this is achieved by increasing the attraction of the $N N$ potential towards a more critical one.

One important result is that the observed increase of the kurtosis is consistent with the early clustering phenomena close to the critical point. The onset of strong $N N$ correlations directly translates into an increase of higher moments. Additional sources of proton fluctuations have not been considered here. While they can further increase the fluctuations to the level of the experimental measurements, we conclude that the effect of correlations should become an important, if not the dominant, signal.

\section{B. Light prenuclei clusters at freeze-out}

In previous sections we have observed that relatively deep nuclear potentials induce nuclear clustering if the system is able to survive for a large amount of time. In this respect, we do not find realistic to search for heavy-fragment production due to critical dynamics, as the required time for their formation is much longer than the typical hadronic stage.

However, within several Fermi/c there is still room for clustering of a few nucleons. In fact, such a mechanism seems to be required, according to Ref. [28], to explain the STAR experimental data. In that reference, the third- and fourth-order cumulants cannot be explained by a model by only nucleon stopping and baryon global conservation. The conclusion of [28] is that some sort of clustering is needed to describe the data. In this work we provide a natural mechanism for clustering if the $N N$ interaction becomes attractive enough close to the critical point.

While for the calculation of the higher-order moments of the proton distribution we have included the contribution of all 

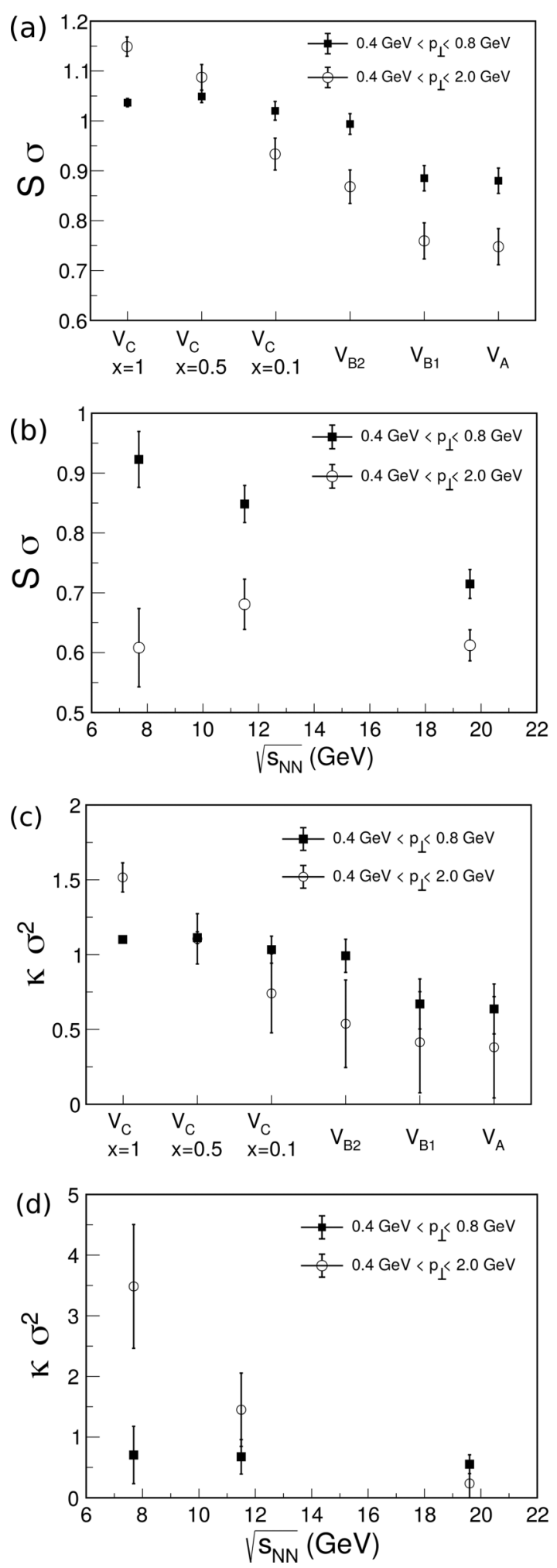

FIG. 23. Scaled skewness (a),(b) and kurtosis (c),(d) as a function of the potential used and from experimental data [5,22] from STAR Collaboration.

protons-within the corresponding kinematic cuts-we will now extract the nuclear clusters which may give rise to light nuclei at post-freeze-out stages. We will also denote these clusters as "prenuclei" and they are products of the nucleon

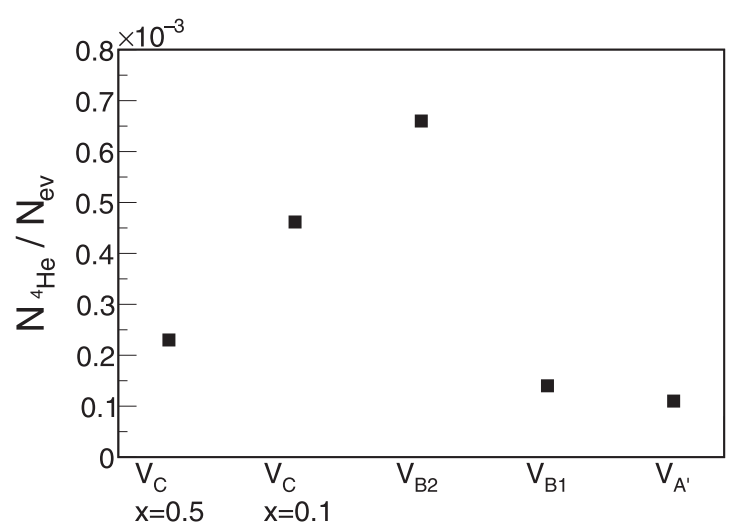

FIG. 24. Multiplicity of pre- ${ }^{4} \mathrm{He}$ (isolated clusters of four nucleons at freeze-out) per event as a function of the $N N$ potential (attraction between $N N$ increases from right to left).

coalescence at freeze-out. If such prenuclei are able to survive as bound objects until the final stage of the fireball at very low temperatures, then they will form states like ${ }^{3} \mathrm{H},{ }^{3} \mathrm{He},{ }^{4} \mathrm{He} . .$. resulting in an excess of light nuclei over the expected thermal production.

For definiteness let us focus on ${ }^{4} \mathrm{He}$, which has a binding energy of $28.3 \mathrm{MeV}$ [29]. Freeze-out temperatures are larger than this energy, but the modification of the nuclear potential can provide extra binding to it. We will look for candidates of ${ }^{4} \mathrm{He}$ nuclei at the final time of our simulation, and search for four nucleons close in phase space at the moment of the freeze-out. If a pair of nucleons are separated by a large distance in the phase space, then they are assumed to not belong to the same cluster. We run our code using several versions of the $N N$ potentials and identify configurations of four nucleons, or "pre- ${ }^{4} \mathrm{He}$." We apply the following criteria:

(1) We only search clusters of four nucleons. If any nucleon also belongs to a different cluster, the whole set is ruled out (as the nucleons belong to bigger nuclei).

(2) The relative position between pairs of nucleons should be small. The rms of ${ }^{4} \mathrm{He}$ is $1.67 \mathrm{fm}$, and the rms for proton is $0.87 \mathrm{fm}$. Assuming a tetrahedron configuration (see Sec. V), one obtains that the distance between the center of two nucleons should be $1.69 \mathrm{fm}$. Giving some freedom to this value due to the thermal motion (deformation of the tetrahedron), we assign a maximal distance of $\Delta r=2 \mathrm{fm}$.

(3) The momenta should also be similar. Taking a typical thermal momentum for ${ }^{4} \mathrm{He}$ of $\sqrt{m T}=0.77 \mathrm{GeV}$, this gives a momentum of $0.11 \mathrm{GeV}$ to each of the Cartesian components of each individual nucleon. We impose the condition that any component of the relative momentum cannot be larger than $\Delta p=0.22 \mathrm{GeV}$.

These two numbers satisfy $\Delta p \Delta r=\mathcal{O}(1)$, so this choice seems natural.

In Fig. 24 we present the number of clusters of four nucleons (pre- ${ }^{4} \mathrm{He}$ ) per event (we use $N_{\mathrm{ev}}=10^{5}$ events for each $N N$ potential). Similarly to the calculation of the moments of the proton distribution, we associate the noncritical potential $V_{A^{\prime}}$ to the experimental collision energy of $\sqrt{s_{N N}}=19.6 \mathrm{GeV}$. 
Then, using this potential in our MD + Langevin code with $N=32$ nucleons, we count the number of such clusters defined by the previous criteria. Notice that in the results of that figure we have not applied the overall scaling factor to the final multiplicity.

The results illustrate the effect of larger clustering formation with the $N N$ potential used. Going from right to left in Fig. 24 we find that the number of pre- ${ }^{4} \mathrm{He}$ increases with the attraction of the nuclear potential. Surprisingly, the most attractive potential $V_{C}$ presents a decrease of the number of clusters. The explanation is that the large attraction binds the nucleon into bigger clusters, i.e., it is more difficult to find four nucleons isolated from the rest.

In spite of the qualitative study we can check that the numbers are not unrealistic. Remember that the $V_{A^{\prime}}$ potential is identified with the collision energy of $\sqrt{s_{N N}}=19.6 \mathrm{GeV}$. The multiplicity of ${ }^{4} \mathrm{He}$ has not been measured at this energy by STAR. However, other light nuclei have been measured by the NA49 experiment [30]. For a close energy of $\sqrt{s_{N N}}=$ $17.3 \mathrm{GeV}\left(E_{\text {beam }}=158 A \mathrm{GeV}\right)$ we know from Ref. [30] that after increasing the mass number in one unit (from $A=1$ to $A=2$, and from $A=2$ to to $A=3$ ), the $d N / d y$ at midrapidity decreases a factor of 100 . Assuming that this scaling holds up to $A=4$ we then expect one nucleus of ${ }^{4} \mathrm{He}$ for each $10^{6}$ nucleons. Applying the same ratio to our simulation, we have that in $N_{\mathrm{ev}}=10^{5}$ events with $N=32$ nucleons we should expect a total of three nuclei of ${ }^{4} \mathrm{He}$. For the $V_{A^{\prime}}$ potential we have numerically obtained 11 pre- ${ }^{4} \mathrm{He}$.

Despite the number of simplifications made in our study, this number seems reasonable due to the fact that only a fraction of prenuclei at freeze-out will eventually populate light nuclei. Our four-nucleon clusters might not necessarily become ${ }^{4} \mathrm{He}$ at the end of the evolution. Assuming a rather sharp freeze-out process, one needs to project the Wigner function of these prenuclei configurations to the actual wave function of ${ }^{4} \mathrm{He}$. This study - which would give a more precise prediction for the produced light nuclei-is left for a future work, and here we restrict ourselves to show the increase of nonequilibrium clusters due to the reduction of the $\sigma$ mass close to $T_{c}$.

\section{SUMMARY AND OUTLOOK}

In this work we have studied baryonic clustering at the freeze-out conditions corresponding to baryon-rich heavy-ion collisions. More specifically, we have observed that both the clustering rate and the properties of the resulting clusters are very sensitive to the magnitude of the effective internucleon potential, and suggest that detailed studies of the baryon distributions will be able to fix such potentials, and ultimately tell us whether the QCD critical point exists or not.

In Sec. II we have defined a set of internucleon effective potentials, which are modifications of the Walecka-Serot model, some with the addition of a long-range component related to massless critical mode at the (hypothetical) critical point. Then in Sec. III we performed some initial studies of baryonic clusters which these potentials can support. The main tool we used is classical molecular dynamics, complemented by a Langevin stochastic force accounting for the mesonic heat bath. For the case of infinite cold nuclear matter, an additional repulsive potential modeling quantum Fermi effects has been used in Appendix E.

If the matter is not exploding and the system evolves long enough, we do observe that the initial stage, with random baryon positions, is always clustering in one or a few large clusters. If the time is not so long, corresponding to $\Delta t \sim 5 \mathrm{fm} / c$ available for the hadronic phase of heavy-ion collisions, the degree of clustering is very strongly dependent on the version of the potential used. Our main result is thus the high sensitivity of this phenomenon to the internucleon potential.

We also tried to imitate an experimental fireball, mapping it to an expanding system. We also impose similar cuts to the experimental acceptance of STAR papers, and calculated the baryon number distribution. We do observe an increase of kurtosis, by about a factor of 3, from the original Walecka potential to our most attractive version. While in this paper we cannot directly compare our results to the STAR BES data, we do focus on one important finding: a growth of the kurtosis of the proton distribution near midrapidity, at the lowest collision energies [5].

Our main qualitative conclusion is that while the evolution time available is insufficient to produce fully developed "nucleosynthesis" with heavy fragments, one definitely should find the baryon distribution in the final state far from thermal equilibrium. Indeed, the confidence in this statement is also provided by similar studies in atomic systems and globular clustering in galaxies (briefly outlined in the corresponding Appendixes). We therefore suggest to look at possible deviations from thermal equilibrium in the yields of light nuclei, such as $d, t,{ }^{3} \mathrm{He},{ }^{4} \mathrm{He}$. A related study in this direction is shown in Refs. [31,32].

Although the specific critical enhancement of the multiparticle fluctuations remains the major goal of this program, one needs to also study other phenomena which can lead to those. In this paper we focused on the clustering of baryons due to their attractive interaction. As we detailed above, significant clustering should in fact occur due to the usual nuclear forces.

\section{ACKNOWLEDGMENTS}

We thank the STAR Collaboration (and X. Luo) for providing the preliminary data of Ref. [5]. We acknowledge R.-A. Tripolt for providing the $\sigma$ spectral functions in [33,34]. This work was supported in part by the U.S. Department of Energy under Contract No. DE-FG-88ER40388.

\section{APPENDIX A: MEAN-FIELD APPROACH TO THE SEROT-WALECKA MODEL}

In this Appendix we remind the reader of a simplified form of nuclear forces, following a model by Serot and Walecka [10]. One important simplifying characteristic is that it only includes the isoscalar mesons: scalar $\sigma$ and vector $\omega$, so there is no difference between coupling to protons and neutrons.

Its Lagrangian density is shown in Eq. (3), and the internucleon potential is written in Eq. (4), which we reproduce here again for convenience,

$$
\tilde{V}_{A}(r)=-\frac{g_{\sigma}^{2}}{4 \pi r} e^{-m_{\sigma} r}+\frac{g_{\omega}^{2}}{4 \pi r} e^{-m_{\omega} r},
$$




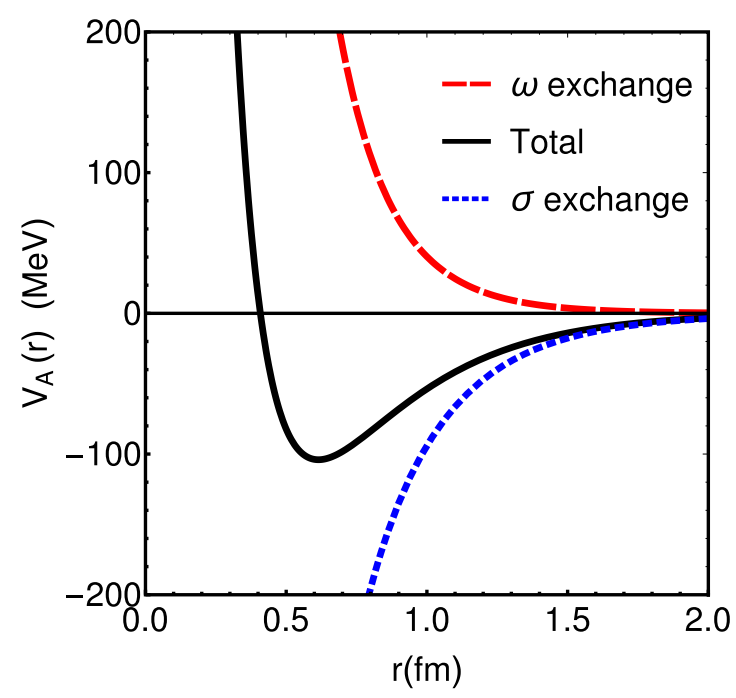

FIG. 25. Solid line: Serot-Walecka potential as given in Eq. (4). Dot-dashed line: Attractive part of the potential given by the first term in Eq. (4). Dashed line: Repulsive contribution of the potential described by the second term of Eq. (4).

with parameters in (5) chosen by mean-field calculations.

In Fig. 25 we illustrate the partial cancellation of the attractive and repulsive terms of this potential.

Considering the case of infinite homogeneous matter of density $n$ and ignoring correlations between the nucleons, one gets the mean potential energy

$$
\langle V\rangle=\frac{n}{2}\left(-\frac{g_{\sigma}^{2}}{m_{\sigma}^{2}}+\frac{g_{\omega}^{2}}{m_{\omega}^{2}}\right) .
$$

If matter is cold, $T=0$, the baryons are in a form of degenerate Fermi gas of quasiparticles with dispersion relation

$$
E_{k}=k+g_{\omega} V_{0}+\sqrt{k^{2}+M_{*}^{2}}, \quad M_{*}=m_{N}-g_{\sigma} \phi_{0} .
$$

Note that if one expands the square root, the leading term of the mean potential $g_{\omega} V_{0}-g_{\sigma} \phi_{0}$ will be the same as the one from the usual nonrelativistic theory, but the kinetic energy term would be $k^{2} / 2 M_{*}$ rather than $k^{2} / 2 m_{N}$. The total energy of the gas is

$$
\begin{aligned}
E_{m f a}= & \frac{g_{\omega}^{2}}{2 m_{\omega}^{2}} n_{B}^{2}+\frac{m_{\sigma}^{2}}{2 g_{s}}\left(m_{N}-M_{*}\right)^{2} \\
& +\frac{\gamma}{(2 \pi)^{2}} \int^{k_{F}} d^{3} k \sqrt{k^{2}+M_{*}^{2}},
\end{aligned}
$$

where the statistical weight $\gamma=4$ for symmetric nuclear matter, and 2 for neutron matter in neutron stars. Two densities, the vector and scalar, can now be written as integrals over the Fermi sphere,

$$
\begin{aligned}
& n_{B}=\frac{\gamma}{(2 \pi)^{2}} \int^{k_{F}} d^{3} k, \\
& n_{s}=\frac{\gamma}{(2 \pi)^{2}} \int^{k_{F}} d^{3} k \frac{M_{*}}{\sqrt{k^{2}+M_{*}^{2}}} .
\end{aligned}
$$

Note that the latter has scalar mass $M_{*}$ in the numerator and the energy in the denominator, which is needed because Lorentz invariant integration measure is $d^{3} k / E_{k}$.

At this stage all is fixed except the scalar mean field (or alternatively $M_{*}$ ): this is a parameter of the homogeneousfield trial function, which, as any variational parameter, should be found from minimization of the ground state energy. This leads to the following equation:

$$
M_{*}=M-\frac{g_{\sigma}^{2}}{m_{\sigma}^{2}} \frac{\gamma}{(2 \pi)^{2}} \int^{k_{F}} d^{3} k \frac{M_{*}}{\sqrt{k^{2}+M_{*}^{2}}},
$$

for $M_{*}$ to be solved numerically. As shown in the original work [10], such a mean-field result can be fitted to reproduce the nuclear matter density and nuclear binding.

For finite spherical nuclei the procedure includes the solution of the mesonic equations of motion,

$$
\begin{aligned}
\left(\partial_{\mu} \partial^{\mu}+m_{\sigma}^{2}\right) \phi & =g_{\sigma} \bar{\psi} \psi, \\
\partial_{\mu} F^{\mu \nu}+m_{\omega}^{2} V^{\nu} & =g_{\omega} \bar{\psi} \gamma^{\nu} \psi,
\end{aligned}
$$

supplemented by Thomas-Fermi-like treatment of baryons. For heavy nuclei the results are rather good.

While this model is only a stripped-down version of nuclear forces and the mean field is only the first of various approximations used for nuclear matter description, we will use it below due to its simplicity. In particular, this model only includes isoscalar exchanges, which means that $p p$ and $p n$ forces are the same. As a result, the only place where isospin matters is in the quantum kinetic energy, since it depends on the number of species. We are however fully aware of the fact that Walecka model parameters are only good for mean-field treatment, and the resulting forces do not describe elastic $N N$ scatterings or the deuteron binding. To improve on this it is possible to increasing the repulsion, via higher $\omega$ coupling $g_{\omega}^{2} \rightarrow 1.4 g_{\omega}^{2}$, so that the resulting potential gets very similar to the Bonn potential [11] (see the right panel of Fig. 1). This is what we will call "modified Walecka potential," which will be used in this work, denoted by $V_{A^{\prime}}$.

\section{APPENDIX B: $\sigma$-MESON DEPENDENCE OF THE $N N$ POTENTIAL FROM THE FUNCTIONAL RENORMALIZATION GROUP}

In Sec. II we have analyzed the modification of the attractive part of the $N N$ potential due to the $\sigma$ mass modification. In this work we have not dealt with the precise dependence of this mass with the temperature/density. This would imply an additional uncertainty dependent on the model used, e.g., linear sigma model, quark-meson model, Nambu-Jona-Lasinio model, etc. On the other hand a more rigorous treatment would involve the modification of the whole spectral function of this state.

In this Appendix we will illustrate how these two issues can be addressed using results of the $\sigma$-meson properties in the $N_{f}=2$ quark-meson model, approached by the application of the functional renormalization group (FRG) [33,34].

The version of the quark-meson model presented in $[33,34]$ contains quarks, antiquarks, pion, and $\sigma$ degrees of freedom. After the evolution of the FRG equations one is able to obtain 
the medium-modified properties of these states in the infrared limit. In particular, the spectral function of the $\sigma$ meson can be obtained at different temperatures and chemical potentials.

In Ref. [33] the critical point of the quark-meson model is located around $T \simeq 10 \mathrm{MeV}$, which seems to be quite low from the phenomenological point of view (this critical temperature is supposed to increase when extending the calculation to $N_{f}=3$ flavors and after introducing the effects of the Polyakov loop potential). Therefore, we will consider two cases: at $T=\mu=0$ where the $\sigma$ screening mass is very close to our vacuum mass $m_{\sigma} \simeq 500 \mathrm{MeV}$ for the potential $V_{A^{\prime}}$, and $T=150 \mathrm{MeV}$ where the $\sigma$ screening mass drops to values around $m_{\sigma} \simeq 280 \mathrm{MeV}$.

The attractive part of the static $N N$ potential is computed as a Fourier transform of the $\sigma$-meson exchange diagram,

$$
V_{\sigma}(\mathbf{r})=g_{\sigma}^{2} \int_{-\infty}^{\infty} d t \int_{-\infty}^{\infty} \frac{d^{4} p}{(2 \pi)^{4}} e^{i p \cdot x} D_{\sigma}^{R}\left(p_{0}, \mathbf{p}\right),
$$

where $p \cdot x \equiv p_{0} t-\mathbf{p} \cdot \mathbf{r}$, and the $\sigma$ retarded propagator is used in the Lehmann representation to account for the complete spectral function $\rho_{\sigma}(\omega, \mathbf{p})$,

$$
D_{\sigma}^{R}\left(p_{0}, \mathbf{p}\right)=-\int_{-\infty}^{\infty} d \omega \frac{\rho_{\sigma}(\omega, \mathbf{p})}{\omega-p_{0}-i \epsilon}
$$

The data from Ref. [34] are given between $\omega \in$ $(-1,1) \mathrm{GeV}$ and $p \in(-1,1) \mathrm{GeV}$. For the case at $T=\mu=$ $0 \mathrm{MeV}$ the $\sigma$-mass pole lies away from the real energy axis (i.e., its real part is above the $\pi-\pi$ unitary threshold). Therefore, the mass appears in the spectral function as a broad pole, which can be numerically integrated in energy and momentum. However, at $T=150 \mathrm{MeV}, \mu=0 \mathrm{MeV}$ the $\sigma$ mass goes down below the unitary threshold and the pole is located on the real axis. In such a case the mass appears in the spectral function as a Dirac delta, which we need to add by hand to the spectral function as the discretized data cannot capture it. Following the conventions in [34] we add to the $\rho_{\sigma}(\omega, \mathbf{p})$ a term like

$$
Z^{-1} \operatorname{sgn}(\omega) \delta\left(\omega^{2}-\mathbf{p}^{2}-m_{\sigma}^{2}\right)
$$

where $m_{\sigma}$ is the pole mass of the $\sigma$ and $Z^{-1}$ is the pole weighting factor (we refer to [34] to see how to compute these from the spectral function).

In Fig. 26 we compare the results coming from the Fourier transform of the spectral function of the $\sigma$ meson, and the simple potential as given in our Eq. (4) with $40 \%$ extra repulsion by the $\omega$ meson $\left(V_{A^{\prime}}\right)$. Even at the quantitative level the two sets of potentials look similar. The main difference of the $\sigma$ potential occurs at small distances, but in this limit the full potential is dominated by the $\omega$ repulsion. In the results using the $\sigma$ spectral function we observe some spurious oscillations around zero, which are nothing but the Gibbs effect coming from the inverse Fourier transform of the spectral function performed within a compact support of energy and momentum.

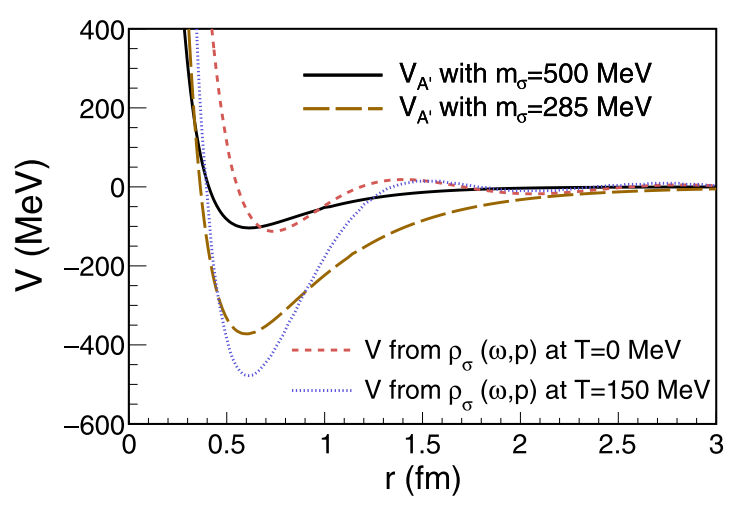

FIG. 26. Solid line: Modified Serot-Walecka potential $V_{A^{\prime}}$ with the form of Eq. (4) and our vacuum parameters. The $\sigma$ mass is $m_{\sigma}=500 \mathrm{MeV}$. Short-dashed line: Result coming from the $\rho$ spectral function in vacuum taken from [34]. The $\sigma$ screening mass in vacuum is $m_{\sigma}=500 \mathrm{MeV}$. Long-dashed line: Modified SerotWalecka potential $V_{A^{\prime}}$ with a $\sigma$ mass of $285 \mathrm{MeV}$, keeping the rest of the parameters as in vacuum. Dotted line: Potential after using the spectral function at $T=150 \mathrm{MeV}, \mu=0$ from [34]. The $\sigma$ screening mass for this temperature is $m_{\sigma}=285 \mathrm{MeV}$.

\section{APPENDIX C: KINETICS AND CLUSTERING IN ATOMIC SYSTEMS}

The simplest atomic systems are those of the noble gases, with spherical atoms and forces depending solely on distances. For a large enough atomic weight, one can neglect quantum effects. For all these reasons, the object of choice is argon, with its $A=40$ (for the most abundant argon isotope) being ten times heavier than ${ }^{4} \mathrm{He}$. By tradition, theoretical studies of it use the simple potential

$$
V(r)=4 \epsilon\left[\left(\frac{\sigma}{r}\right)^{12}-\left(\frac{\sigma}{r}\right)^{6}\right] .
$$

Its minimum is at $V\left(2^{1 / 6} \sigma\right)=-\epsilon$. Following one of the classic MD simulations from the 1960s [35], one can use parameter values $\sigma=3.4 \AA, \epsilon=120 \mathrm{~K}$.

The shapes of this potential and that of the nuclear forces (Walecka model) are compared in Fig. 27. It shows that Lennard-Jones potential is much more narrow. The ratio of the potential to the temperature is similar to the problem we study, provided the temperature of argon is $T \sim 100 \mathrm{~K}$.

The work [35] focused on one temperature $T=94 \mathrm{~K}$ and one density $\rho=1.37 \mathrm{~g} / \mathrm{cm}^{3}$, which is well in the liquid phase. We minimally modify our MD (without Langevin dynamics) to run an isocanonical simulation (by rescaling of instantaneous temperature). We use $N=108$ and similar conditions with a reduced temperature of $T^{*}=T / \epsilon=0.783$ and a reduced density of $n^{*}=N / V \sigma^{3}=0.814$. The radial two-body correlation function $g(r)$ is shown in the upper panel of Fig. 28, presenting several peaks, indicating strong correlations between the atoms at particular distances.

Increasing the density, one crosses the phase transition to a solidlike phase. A new simulation with $n^{*}=1.1$ gives the radial distribution function in the lower panel of Fig. 28. The amount of very pronounced peaks is a signature of the solid (crystalline) structure of the system. In this case the 


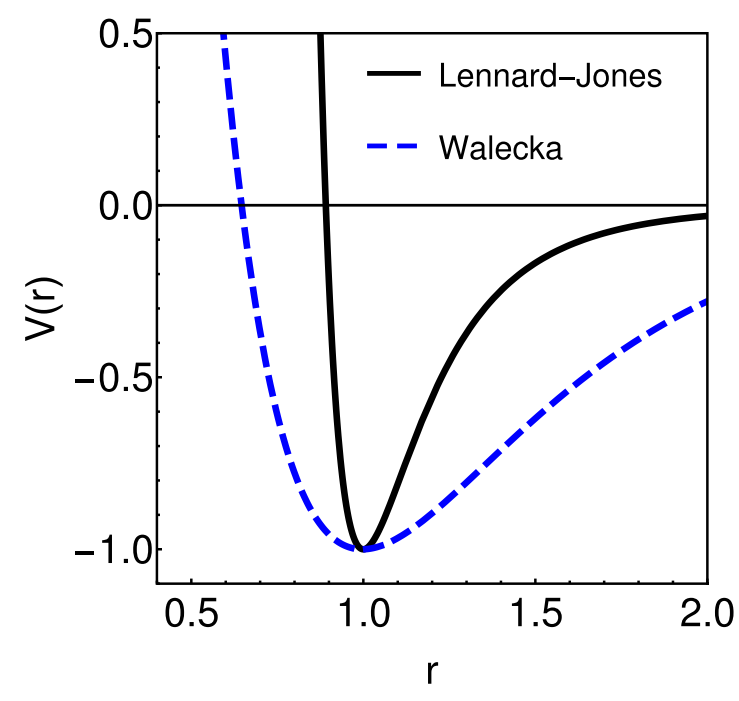

FIG. 27. Similarly normalized Lennard-Jones (black solid) and Walecka (blue dashed) potentials.

distribution of peaks can be identified with a face-centeredcubic distribution (which is the configuration used to initialize our simulation).

The standard MD simulation, unlike Monte Carlo ones, have not just static (fixed time) but also the time-dependent information, such as velocity-velocity and other correlation functions, Using standard Green-Kubo formulas one can calculate diffusion constant, viscosity, etc. We however would not go into vast literature on the kinetic properties, except to note that liquid argon, like other liquids, has a secondorder critical point, and studies of the singularities of kinetic coefficients there remain to be better understood.

Finally, we would like to mention instead a particular large-scale MD simulation [36], using as many as a billion atoms, and focusing on transition from homogeneous particle distributions to liquid phase, at supersaturated conditions. As is well known, the process can be divided into two stages: (i) creation of critical clusters, with $i^{*}$ particles in them; and (ii) their subsequent linear growth as a function of time with a certain rate. The large scale of the simulation had allowed one to cover a range of temperatures and densities, in which the clustering rates change over many decades, and cluster sizes grow to well over 100 particles. However, what is most important is that in all cases the critical clusters are relatively small, ranging from $i^{*} \sim 12$ to about 100 atoms. Therefore, the classical theory of nucleation-treating these clusters as macroscopic drops with a surface and volume free energies-needs to be corrected. After the actual energies of these clusters are used, the corrected theory was shown to work well. Equilibrium configuration of small and medium size clusters in Lennard-Jones interaction has been studied, e.g., in [37].

\section{APPENDIX D: GLOBULAR CLUSTERS IN GALAXIES}

Gravity is the simplest attractive interaction, and the stars in the galaxy-which can be well approximated by structureless point masses - are the simplest classical objects one
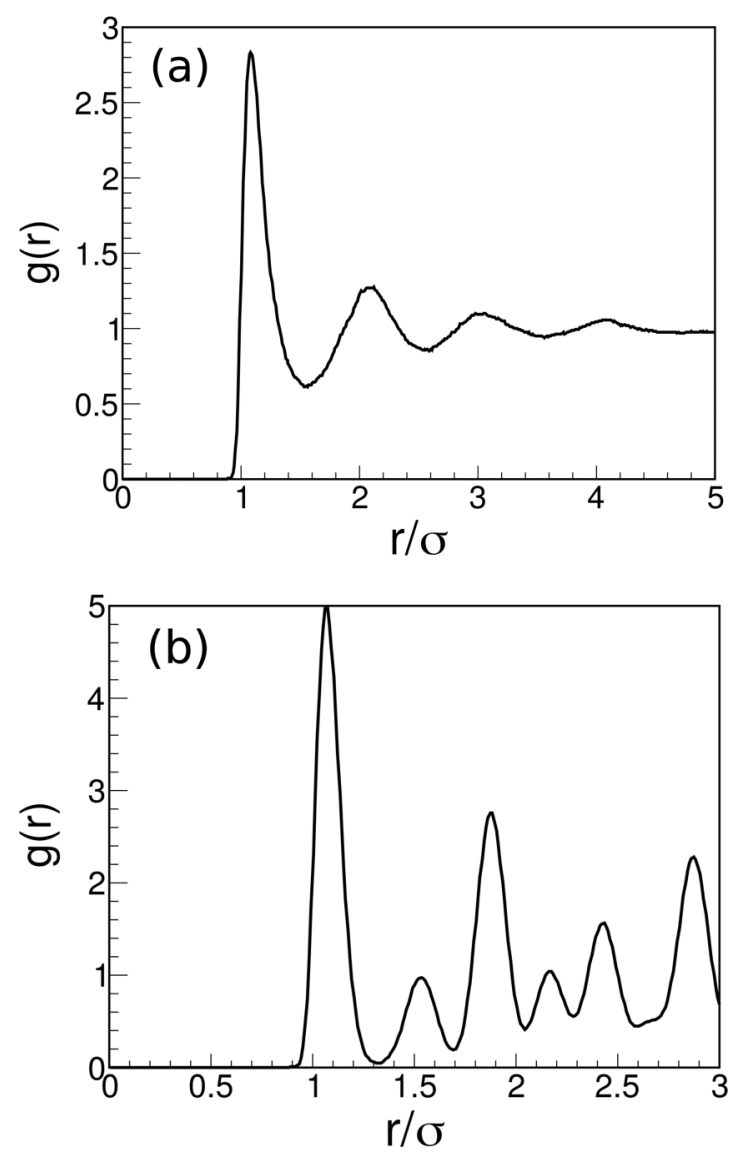

FIG. 28. Two-body correlation function for argon from MD simulations with $N=108$ particles. (a) $T^{*}=T / \epsilon=0.783, n^{*}=$ $N / V \sigma^{3}=0.814$ (liquid phase). (b) $T^{*}=0.783, n^{*}=1.1$ (solid phase).

can think of. Galaxies themselves and the globular clusters are products of instabilities induced by long-range attractive interaction, and all of them appear from the homogeneous cosmological plasma at a certain temperature.

We will not be discussing here those instabilities and complicated paths which lead to globular cluster formation, focusing at the classical theory of quasistationary clusters. Since this field belongs to astronomy and is rather far from nuclear physics, we include in this summary its main elements.

Globular clusters are approximately spherically symmetric bound states of many stars. Their typical number $N$ varies from $10^{3}$ to $10^{6}$, which is much smaller than that in the whole galaxies $\sim 10^{11}$. For definiteness, we will mention numbers for $N=10^{5}$. The clusters are believed to possess black holes at their centers, intermediate in mass between those due to a star collapse and those at the centers of the galaxies. In any case, their masses are way too small to play any role in what follows.

The main parameters of the clusters can be inferred from their size, $\sim 10 \mathrm{pc}$, and the typical velocity, $v \approx 10 \mathrm{~km} / \mathrm{s}$, resulting in the smallest of relevant time scales, the crossing time,

$$
t_{\text {crossing }}=\frac{r}{v} \sim 10^{6} \mathrm{yr} .
$$


Scattering leads to equilibration of the system, relaxing it to certain virial equilibrium in which we see the observed clusters. The relaxation time of a cluster is

$$
t_{\text {relaxation }} \sim 10^{9} \text { yr. }
$$

This equilibrium is however a quasiequilibrium, since collisions make a small fraction of the stars venture above the escape velocity and leave the cluster. The largest time scale is called the "evaporation time" (assuming cluster is not surrounded by any matter) which is

$$
t_{\text {evaporation }} \sim 10^{10} \mathrm{yr} .
$$

It qualitatively coincides with the age of observed clusters and the lifetime of the Universe.

Considering an object with a unit mass, we define its energy by

$$
\epsilon=-\frac{v^{2}}{2}-\Phi(r)+\Phi_{0}
$$

Note the minus signs compared to the usual definition: so positive $\epsilon$ corresponds to binding. The gravitational potential at distance $r$ from the center $\Phi(r)$ is, as usual, defined up to a constant, which we will select later. Note that $\epsilon=0$ defines the (coordinate dependent) escape velocity $v_{e}=$ $\sqrt{-2 \Phi(r)+2 \Phi_{0}}$.

Step 1 is to satisfy the stationary Boltzmann equation for the star distribution function $f(\vec{x}, \vec{v})$. Setting $\partial f / \partial t=0$ and neglecting the collision term, one has

$$
\left(\vec{v} \cdot \vec{\nabla}_{x}\right) f-\left(\vec{\nabla}_{x} \Phi\right) \cdot \frac{\partial f}{\partial \vec{v}}=0 .
$$

This however is achieved rather easily, for any distribution of the form $f(\epsilon(\vec{x}, \vec{v}))$.

Step 2 is the selection of a particular distribution of such kind. We will discuss the so-called King distribution, in which $f=0$ for negative $\epsilon$ values (that is, the cluster has no unbound stars), and for positive $\epsilon$ it is

$$
f_{K}(\epsilon)=\text { const }\left(2 \pi \sigma^{2}\right)^{-3 / 2}\left[e^{\epsilon / \sigma^{2}}-1\right],
$$

which is a shifted Maxwell-Boltzmann distribution with temperature $T=\sigma^{2}$.

Step 3 is a calculation of the corresponding density of stars, which includes the integration over the velocity. Note that it is limited by the escape velocity defined via the potential, so the density obtained is the function of the potential $\psi=\Phi-\Phi_{0}$,

$$
\begin{aligned}
\rho_{K}(\psi) & =\frac{\text { const }}{\left(2 \pi \sigma^{2}\right)^{3 / 2}} \int_{0}^{\sqrt{2 \psi}}\left(e^{\left(\psi-v^{2} / 2\right) / \sigma^{2}}-1\right) d^{3} v \\
& =-\frac{4}{3 \sqrt{\pi}} \frac{\sqrt{\psi}}{\sigma}\left(\frac{\psi}{\sigma^{2}}+\frac{3}{2}\right)+e^{\psi / \sigma^{2}} \operatorname{Erf}\left(\frac{\sqrt{\psi}}{\sigma}\right) .
\end{aligned}
$$

This complicated function is plotted in Fig. 29, and one can see that it is a monotonously rising one.

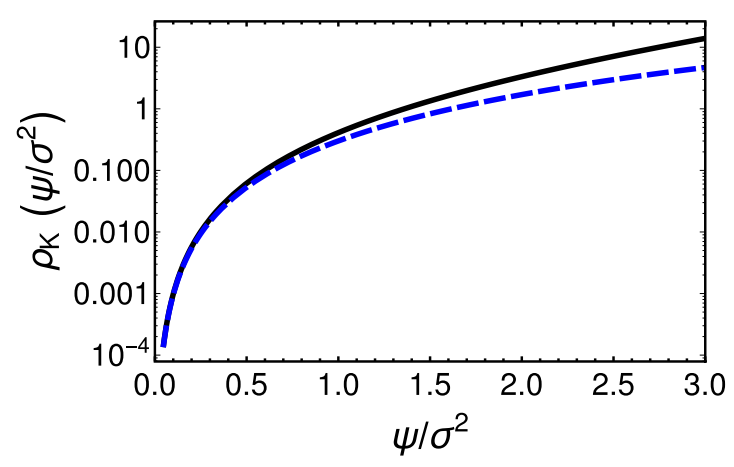

FIG. 29. The function $\rho_{K}\left(\psi / \sigma^{2}\right)$ defined in Eq. (D7) is shown by the black solid line, together with its asymptotic form at small values of the argument $0.30\left(\psi / \sigma^{2}\right)^{5 / 2}$, shown by the blue dashed line.

The density is the source of the potential itself, so now we come across the main dynamical equation to be solved, the Poisson equation for the potential. In case of spherical symmetry it is

$$
\frac{1}{r^{2}} \frac{d}{d r}\left(r^{2} \frac{d \psi}{d r}\right)+4 \pi G_{N} \rho_{K}[\psi(r)]=0,
$$

which can be solved numerically starting from the center. The value $\psi(0)$ is the single input parameter, the derivative needs to be vanishing at the center $\psi^{\prime}(0)=0$. The solution can be followed until the point where $\psi=0$ : and as is clear from the expression above for the density, at that point the density vanished as well since the integration region till the escape velocity shrinks to zero. An example of the resulting potential after solving (D8) is shown in Fig. 30. Substituting the resulting $\psi(r)$ into the universal $\rho(\psi)$ one finally obtains the spatial distribution of the stars in the cluster.

\section{APPENDIX E: COLD NUCLEAR MATTER AND QUANTUM FERMI REPULSION}

To account for quantum repulsion in the simulations, the simplest thing one can do is add the Fermi energy, evaluated in the spirit of the Thomas-Fermi approach from the density profile, to the classical kinetic and potential energy.

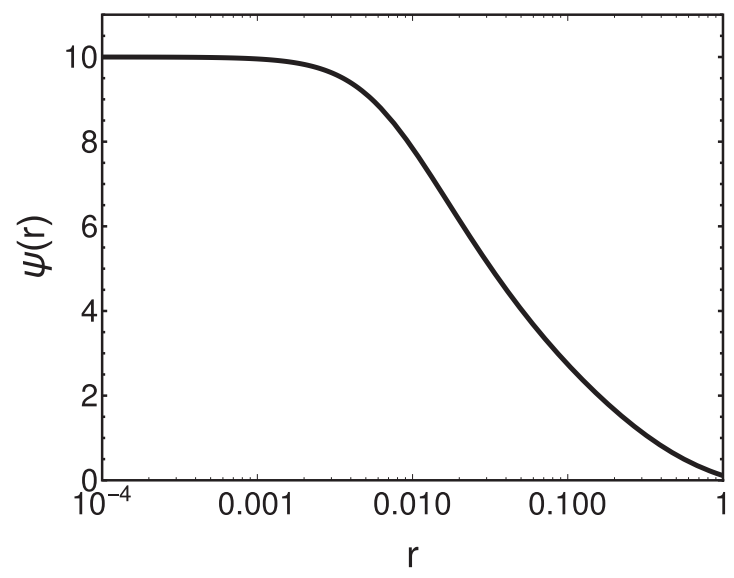

FIG. 30. The solution of the Poisson equation with the source $\rho_{K}(\psi), \psi(r)$ vs $r$ at $G_{N} \times$ const $=1, \sigma=1$, and $\psi(0)=10$. 
A full account for both quantum and thermal fluctuations can be done in approaches called "quantum open systems"; see, e.g., Ref. [38] for its application to motion and heavy quarks and quarkonia, as well as general references.

Strictly speaking, a complete account for quantum effects would require going from classical molecular dynamics to full path integrals. As is well known, while for distinguishable particles and bosons it can be considered to be just a technical complication, for fermions the amplitude needs to be antisymmetrized, which brings in the notorious sign problem. The effective Fermi repulsion, acting as a kind of repulsive potential, generates correlations between particles which depend strongly on their mutual distance.

In the 1980s Zhirov and one of us studied paths of fermions moving in one dimension. This case is special because one can always enumerate fermions along the line, and thus pretend that the "exchange" never happens. For a small time step $t_{a}$ the one-particle amplitude is

$$
U\left(x_{f}, x_{i}, t_{a}\right) \sim \exp \left[-\frac{m\left(x_{f}-x_{i}\right)^{2}}{2 t_{a}}-t_{a} V\left(\frac{x_{f}+x_{i}}{2}\right)\right],
$$

and for two particles it can be written as

$$
\begin{aligned}
& U\left(x_{1}^{f}, x_{1}^{i}, t_{a}\right) U\left(x_{2}^{f}, x_{2}^{i}, t_{a}\right)-U\left(x_{1}^{f}, x_{2}^{i}, t_{a}\right) U\left(x_{2}^{f}, x_{1}^{i}, t_{a}\right) \\
& \quad \approx U\left(x_{1}^{f}, x_{1}^{i}, t_{a}\right) U\left(x_{2}^{f}, x_{2}^{i}, t_{a}\right) \exp \left[-t_{a} V_{\text {Pauli }}\right],
\end{aligned}
$$

with the "Pauli potential" defined as

$$
V_{\text {Pauli }}=-\frac{1}{t_{a}} \ln \left[1-\exp \left(\frac{-m\left(x_{1}^{f}-x_{2}^{f}\right)\left(x_{1}^{i}-x_{2}^{i}\right)}{t_{a}}\right)\right] \text {. }
$$

Note that when two particles get close, the exponent becomes close to 1 , the argument of the $\log$ near zero, and the potential gets very high. So, a node of the amplitude can be viewed as a repulsive potential. This is going in the right direction: indeed, fermions must have a larger energy than distinguishable particles in the same setting. If particles never jump over the note-generated barrier, their order along the line remains preserved, and if the Pauli potential is included, the simulation

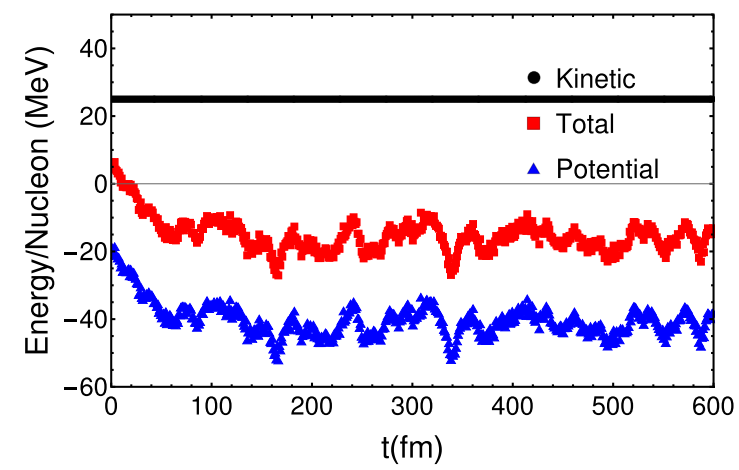

FIG. 31. Kinetic (black circles), total (red squares), and potential (blue triangles) energies per nucleon vs time in the infinite matter calculation. In addition to the Walecka $V_{A^{\prime}}$ we implement the localization potential in Eq. (E4) with $a=0.75 \mathrm{fm}^{3}$. can be done by traditional Monte Carlo. We checked it for several $(n=3-5)$ particles put in a harmonic potential: for distinguishable particles the ground state energy is $\hbar \omega(1 / 2+$ $1 / 2+\cdots)$ but for fermions it should be $\hbar \omega(1 / 2+3 / 2+$ $5 / 2+\cdots)$ since each must be put into the next available level. So, our algorithm with this "Pauli potential" worked correctly. The work was concluded in Ref. [39]. A description of the method and its usage is in Ref. [40], in which many tests have been successfully performed.

The next step forward, allowing to use this idea in any dimension, was made by Ceperley [41]. It has been applied to fermionic problems, including liquid ${ }^{3} \mathrm{He}$. The main idea can be explained if one considers various paths of one fermion, keeping all other fermion paths frozen. The one-dimensional node of the amplitude gets promoted to a "nodal surface," which surrounds each fermion, keeping it inside a "nodal cell." Paths which are not allowed to leave the nodal cell are called "restricted": the sum over the restricted paths obviously has no sign change.
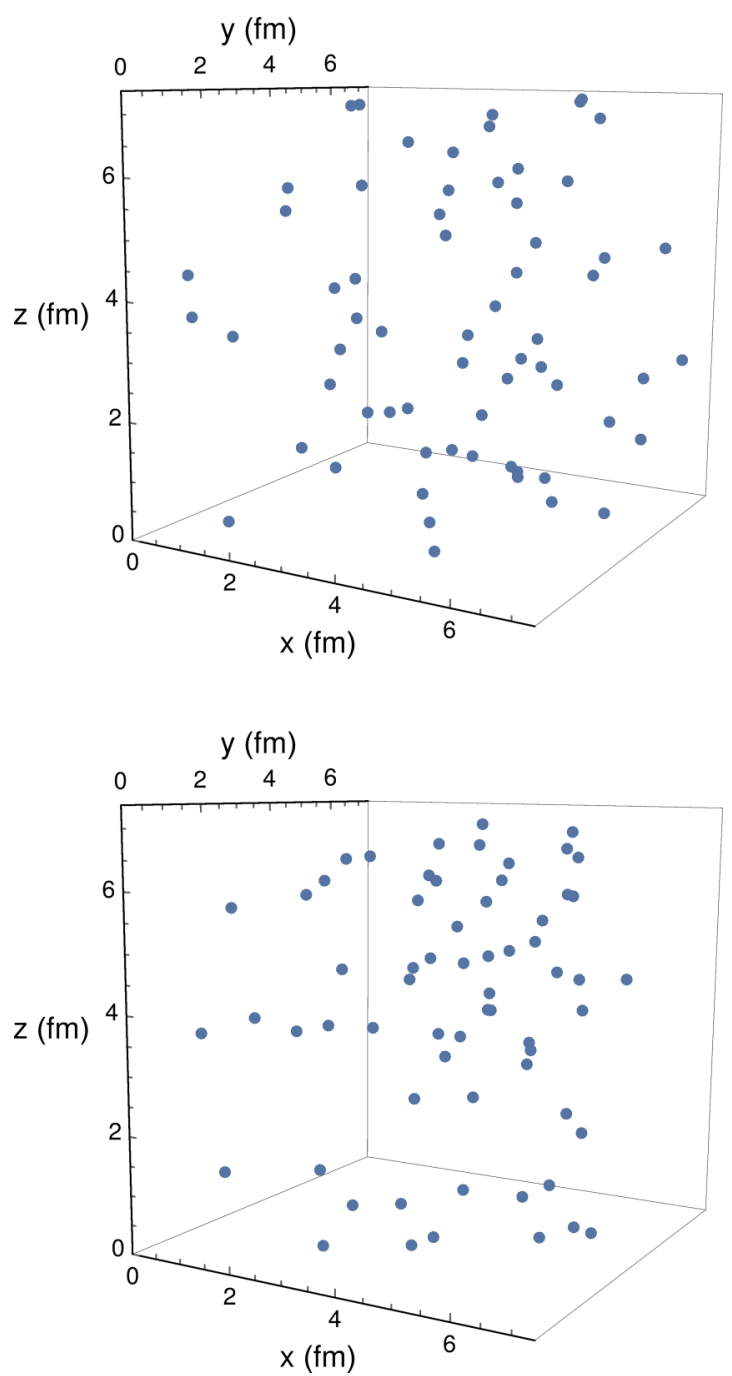

FIG. 32. Initial configuration (top) of nucleons in coordinate space and configuration at an arbitrary time after equilibration (bottom). 
The nodal surface model corresponds to a certain constant potential well with the location of the wall depending on those of other particles. The radius of this surface can be tuned to reproduce the Fermi energy of an ideal gas.

Instead of a sharp wall we decided to include a more smooth localization potential, of the form

$$
V_{\mathrm{loc}}\left(x_{i j}\right)=a \frac{\hbar^{2}}{m_{N} x_{i j}^{5}},
$$

where the exponent is chosen rather arbitrary as long as Pauli repulsion at short distances is achieved.

To normalize this effective potential we attempted to simulate properties of cold homogeneous nuclear matter by our molecular dynamics scheme,

$$
\begin{aligned}
\frac{d \vec{x}_{i}}{d t} & =\frac{\vec{p}_{i}}{m_{N}}, \\
\frac{d \vec{p}_{i}}{d t} & =-\sum_{j \neq i} \frac{\partial V\left(\left|\vec{x}_{i}-\vec{x}_{j}\right|\right)}{\partial \vec{x}_{i}},
\end{aligned}
$$

where $V$ represents the pairwise potential, the sum of the localization potential plus one among the different possibilities described in the main text. We use the Walecka potential $V_{A^{\prime}}(r)$ with increased repulsion, which is closer to the $N N$ phenomenological potentials for nuclear matter. To simulate an infinite system we work on a cubic box with periodic boundary conditions. In such a box the particle density is fixed to the nuclear density at saturation $n_{0}=0.16 \mathrm{fm}^{-3}$ with $N=64$ the total number of nucleons. To account for the interactions of the particles in the box and those outside, we use the method of images, where in the sum of Eq. (E5) we consider the contributions from all $j$ particles within a number of copies of the box in each spatial direction (positive and negative). The number of images (or copies of the elementary box) per each direction is set to 2 .

After a transient regime, the MD simulation reaches an equilibrium state with constant potential and kinetic energies [with statistical fluctuations of $\mathcal{O}(1 / \sqrt{N})$ ]. For infinite nuclear matter at saturation an average Fermi momentum of $p_{F} \sim 260 \mathrm{MeV}$ translates into a kinetic energy per nucleon of $K / N \approx 25 \mathrm{MeV}$. Lacking of quantum dynamics in the classical MD we achieve this value of $K / N$ by forcing a isokinetic simulation by rescaling the velocity of each particle by $\sqrt{K / K_{\text {inst }}}$, where $K_{\text {inst }}$ is the instantaneous value of the kinetic energy at a given time step.

The expected energy per nucleon at saturation $E / N=$ $-16 \mathrm{MeV}$ provides the additional constraint that helps us to fix the remaining parameter of the simulation, the strength of the localization potential, to $a=0.75 \mathrm{fm}^{3}$. The resulting energies versus time are given in Fig. 31. After the equilibration time $(\sim 100 \mathrm{fm} / c)$ we can measure the average total energy (binding energy) per nucleon. We obtain $-16.6 \mathrm{MeV}$, a fair value for our illustrative purposes. For dedicated computations a more precise value of $a$ can be extracted, using more nucleons in the simulation in order to reduce the statistical fluctuations of $E / N$ (going as $1 / \sqrt{N}$ ).

We find a rather homogeneous system at equilibrium with evidence of a slight grouping of nucleons. In the upper panel of Fig. 32 we show the initial configuration of nucleons at random positions in a volume of $(7.37 \mathrm{fm})^{3}$. In the lower panel we show the spatial configuration of the nucleon for an arbitrary time well after the equilibration time.

Quantum effects via localization potential - important for a $T \simeq 0$ calculation-will be absent around the freeze-out temperatures, where kinetic energy is expected to be dominated by thermal fluctuations.
[1] A. Andronic, P. Braun-Munzinger, K. Redlich, and J. Stachel, Nature 561, 321 (2018).

[2] J. P. Bondorf, A. S. Botvina, A. S. Ilinov, I. N. Mishustin, and K. Sneppen, Phys. Rep. 257, 133 (1995).

[3] M. Stephanov, K. Rajagopal, and E. Shuryak, Phys. Rev. Lett. 81, 4816 (1998).

[4] M. Stephanov, K. Rajagopal, and E. Shuryak, Phys. Rev. D 60, 114028 (1999).

[5] X. Luo (STAR Collaboration), PoS (CPOD2014) 019 (2015).

[6] R. Arnaldi et al. (NA60 Collaboration), Phys. Rev. Lett. 96, 162302 (2006).

[7] R. Rapp and J. Wambach, Adv. Nucl. Phys. 25, 1 (2002).

[8] J. R. Pelaez, Phys. Rep. 658, 1 (2016).

[9] L. Y. Glozman, C. B. Lang, and M. Schröck, Phys. Rev. D 86, 014507 (2012).

[10] B. D. Serot and J. D. Walecka, Adv. Nucl. Phys. 16, 1 (1986).

[11] R. Machleidt, Phys. Rev. C 63, 024001 (2001).

[12] N. Ishii, S. Aoki, and T. Hatsuda, Phys. Rev. Lett. 99, 022001 (2007).

[13] B. A. Gelman, E. V. Shuryak, and I. Zahed, Phys. Rev. C 74, 044909 (2006).

[14] J. J. Thomson, Philos. Mag. S. 7, 237 (1904).
[15] A. Bazavov et al., Phys. Rev. D 95, 054504 (2017).

[16] A. Bazavov et al. (HotQCD Collaboration), Phys. Rev. D 96, 074510 (2017).

[17] J. Aichelin and H. Stoecker, Phys. Lett. B 176, 14 (1986)

[18] G. Peilert, J. Randrup, H. Stoecker, and W. Greiner, Phys. Lett. B 260, 271 (1991).

[19] N. Sasaki, O. Miyamura, S. Muroya, and C. Nonaka, Phys. Rev. C 62, 011901 (2000).

[20] P. Kovtun, D. T. Son, and A. O. Starinets, J. High Energy Phys. 10 (2003) 064.

[21] L. Adamczyk et al. (STAR Collaboration), Phys. Rev. C 96, 044904 (2017).

[22] L. Adamczyk et al. (STAR Collaboration), Phys. Rev. Lett. 112, 032302 (2014).

[23] W. Llope, PoS (CPOD2017) 010 (2018).

[24] Y. B. Ivanov and A. A. Soldatov, Phys. Rev. C 97, 024908 (2018).

[25] F. Karsch and K. Redlich, Phys. Lett. B 695, 136 (2011).

[26] X. Luo, J. Phys. G 39, 025008 (2012).

[27] If we had simulated a total number of protons of $N=32 \times$ $6.6=211$, then we would not need to perform this overall 
scaling. However, $N=32$ allows for an efficient and less timeconsuming computation.

[28] A. Bzdak, V. Koch, and V. Skokov, Eur. Phys. J. C 77, 288 (2017).

[29] M. Wang, G. Audi, F. G. Kondev, W. J. Huang, S. Naimi, and X. Xu, Chin. Phys. C 41, 030003 (2017)

[30] T. Anticic et al. (NA49 Collaboration), Phys. Rev. C 94, 044906 (2016).

[31] K.-J. Sun, L.-W. Chen, C. M. Ko, and Z. Xu, Phys. Lett. B 774, 103 (2017).

[32] K.-J. Sun, L.-W. Chen, C. M. Ko, J. Pu, and Z. Xu, Phys. Lett. B 781, 499 (2018).
[33] R.-A. Tripolt, N. Strodthoff, L. von Smekal, and J. Wambach, Phys. Rev. D 89, 034010 (2014).

[34] R. A. Tripolt, Ph.D. thesis, TU Darmstadt, 2015.

[35] A. Rahman, Phys. Rev. 136, A405 (1964).

[36] J. Diemand, R. Angelil, K. K. Tanaka, and H. Tanaka, J. Chem. Phys. 139, 074309 (2013).

[37] M. R. Hoare and P. Pal, Nat. Phys. Sci. 230, 5 (1971).

[38] C. Young and K. Dusling, Phys. Rev. C 87, 065206 (2013).

[39] O. O. Tursunov and O. V. Zhirov, Phys. Lett. B 222, 110 (1989).

[40] B. L. G. Bakker, M. I. Polikarpov, and A. I. Veselov, Few Body Syst. 25, 101 (1998).

[41] D. M. Ceperley, Phys. Rev. Lett. 69, 331 (1992). 